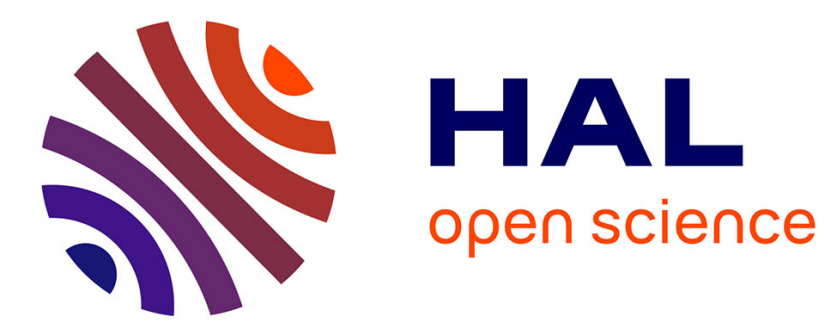

\title{
Tidally-forced turbulence in planetary interiors
}

\author{
Alexander Grannan, Benjamin Favier, Michael Le Bars, Jonathan M. Aurnou
}

\section{To cite this version:}

Alexander Grannan, Benjamin Favier, Michael Le Bars, Jonathan M. Aurnou. Tidally-forced turbulence in planetary interiors. Geophysical Journal International, 2016, 208 (3), pp.1690-1703. 10.1093/gji/ggw479 . hal-01478990

\section{HAL Id: hal-01478990 https://hal.science/hal-01478990}

Submitted on 25 Oct 2018

HAL is a multi-disciplinary open access archive for the deposit and dissemination of scientific research documents, whether they are published or not. The documents may come from teaching and research institutions in France or abroad, or from public or private research centers.
L'archive ouverte pluridisciplinaire $\mathbf{H A L}$, est destinée au dépôt et à la diffusion de documents scientifiques de niveau recherche, publiés ou non, émanant des établissements d'enseignement et de recherche français ou étrangers, des laboratoires publics ou privés. 


\title{
Tidally forced turbulence in planetary interiors
}

\author{
A.M. Grannan, ${ }^{1,2}$ B. Favier, ${ }^{2}$ M. Le Bars ${ }^{2}$ and J.M. Aurnou ${ }^{1}$ \\ ${ }^{1}$ Department of Earth, Planetary, and Space Sciences, University of California-Los Angeles, Los Angeles, CA, USA. E-mail: agrannan@ucla.edu \\ ${ }^{2}$ Aix Marseille Univ, CNRS, Centrale Marseille, IRPHE, Marseille, France
}

Accepted 2016 December 21. Received 2016 December 13; in original form 2016 April 27

\begin{abstract}
SUMMAR Y
The turbulence generated in the liquid metal cores and subsurface oceans of planetary bodies may be due to the role of mechanical forcing through precession/nutation, libration, tidal forcing and collisions. Here, we model the response of an enclosed constant density fluid to tidal forcing by combining laboratory equatorial velocity measurements with selected highresolution numerical simulations to show, for the first time, the generation of bulk filling turbulence. The transition to saturated turbulence is characterized by an elliptical instability that first excites primary inertial modes of the system, then secondary inertial modes forced by the primary inertial modes, and then bulk filling turbulence. The amplitude of this saturated turbulence scales with the body's elliptical distortion, $U \sim \beta$, while a time- and radially averaged azimuthal zonal flow scales with $\beta^{2}$. The results of the current tidal experiments are compared with recent studies of the libration-driven turbulent flows studied by Grannan et al. and Favier et al. Tides and libration correspond to two end-member types of geophysical mechanical forcings. For satellites dominated by tidal forcing, the ellipsoidal boundary enclosing the internal fluid layers is elastically deformed while, for librational forcing, the core-mantle boundary possesses an inherently rigid, frozen-in ellipsoidal shape. We find striking similarities between tidally and librationally driven flow transitions to bulk turbulence and zonal flows. This suggests a generic fluid response independent of the style of mechanical forcing. Since $\beta \lesssim 10^{-4}$ in planetary bodies, it is often argued that mechanically driven zonal velocities will be small. In contrast, our linear scaling for mechanically driven bulk turbulence, $U \sim \beta$, suggests geophysically significant velocities that can play a significant role in planetary processes including tidal dissipation and magnetic field generation.
\end{abstract}

Key words: Numerical solutions; Instability analysis; Tides and planetary waves; Core, outer core and inner core; Planetary interiors.

\section{INTRODUCTION}

Observations made from Earth and from spacecraft missions suggest the presence of liquid metal cores in terrestrial bodies like Mercury (Stark et al. 2015), Mars (Yoder et al. 2003), Io (Anderson et al. 1996) and Ganymede (Schubert et al. 2004), as well as subsurface oceans in Europa (Anderson et al. 1998), Ganymede (Schubert et al. 2004) and Enceladus (Cadek et al. 2016; Thomas et al. 2016). Gravitational interactions between a variety of celestial bodies, from stars and planets to satellites and asteroids, can periodically perturb both a body's shape and the direction and magnitude of its rotation vector. Such perturbative effects can generate mechanical forcing of interior fluid motions through libration, tidal deformation and precession/nutation (Comstock \& Bills 2003; Van Hoolst et al. 2013).

Paleomagnetic measurements of rock samples from smaller terrestrial bodies reveal the remnant signatures of self-generated dynamo fields (e.g. Garrick-Bethell et al. 2009; Fu et al. 2012; Tarduno et al. 2012; Johnson et al. 2015). The assumed driver for the fluid motions responsible for dynamo generation is thermo-compositional convection (e.g. Jones 2011; Schubert \& Soderlund 2011; O'Rourke \& Stevenson 2016). However, the existence of dynamos on smaller bodies is difficult to reconcile with our current understanding of the conditions necessary for magnetic field generation in terrestrial bodies through thermo-compositional convection alone (e.g. Nimmo 2009; Pozzo et al. 2012; O1son 2013; Zhang et al. 2015). Thus, recent numerical studies, (e.g. Tilgner 2005; Wu \& Roberts 2009, 2013; Cébron \& Hollerbach 2014; Wei et al. 2014) have begun to address how mechanical forcing can also drive dynamos by injecting a portion of the vast quantity of rotational energy from the primary-satellite orbital systems into the interior fluid motions. However, even the basic properties of turbulence generated by mechanical forcing are not yet well characterized.

The current work focuses on comparing new results from purely hydrodynamic analogue models of tidal forcing in an ellipsoidal container with previous studies of longitudinal librational forcing, referred hereafter as librational forcing. In geophysical terms, theses analogue models replicate the tidal and librational forcing in deformed bodies as shown in polar view in Figs 1(a) and (b), 
(a) Tides

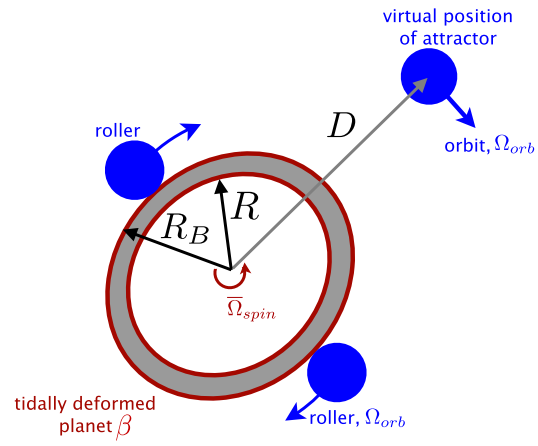

(b) Libration

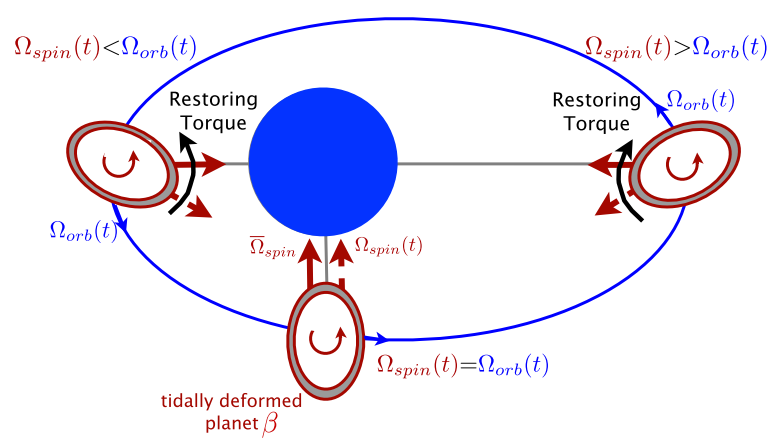

Figure 1. (a) A polar view of the model for a tidally deformed elastic body of amplitude $\beta$ with a fluid interior spinning at $\bar{\Omega}_{\text {spin }}$. A virtual attractor orbiting at $\Omega_{\text {orbit }}(t)$ is simulated in experiments by using two symmetric rollers. The deformed body's r.m.s radius is $R_{B}$, the fluid radius is $R$ and the average distance between the two bodies is $D$. (b) A polar view of the model for longitudinal libration of a rigid synchronous planet with a fluid interior rotating at $\Omega_{\text {spin }}(t)$, and orbiting at $\Omega_{\text {orbit }}(t)$ where, when time-averaged, $\bar{\Omega}_{\text {spin }}=\bar{\Omega}_{\text {orbit. }}$. The solid red arrow denotes the direction toward the gravitational partner. The dashed red arrow denotes the direction of the long axis of the deformed ellipsoid. The misalignment between the two red arrows leads to restoring torques (black arrow) that oscillate the deformed boundary. Schematics adapted from Le Bars et al. (2010), Noir et al. (2012).

respectively. For tides and libration, the fluid enclosed by the greycoloured ellipsoidal shell is constrained by a time-averaged angular rotation rate, $\bar{\Omega}_{\text {spin }}$. In Fig. 1(a), a planet having low rigidity and hence a high tidal potential Love number $k_{2}$, is deformed by an orbiting attractor (e.g. Greff-Lefftz et al. 2005). As such, the planet and boundary deformation rotate at separate rates $\bar{\Omega}_{\text {spin }}$ and $\Omega_{\text {orb }}$, respectively.

In librational forcing of a rigid (low $k_{2}$ ), synchronized planet, the time-averaged spin and orbital rates are equal, $\bar{\Omega}_{\text {spin }}=\bar{\Omega}_{\text {orb }}$. The model for this librational forcing is shown schematically in Fig. 1(b) The eccentricity of the orbit leads to variations in the orbital rate following Kepler's third law and creates a phase lag between the equatorial bulge and the line connecting the centres of mass for the two bodies as shown by the dashed and solid red arrows in Fig. 1(b), respectively. Periodic torques, shown as black arrows, are induced to restore this alignment, leading to oscillations in the rotation rate, $\Omega_{\text {spin }}(t)$, of the deformed boundary about the average spin rate $\bar{\Omega}_{\text {spin }}$.

Mechanical forcing through tides and libration is capable of generating laminar and turbulent fluid motions in the bulk of the fluid. It has been well-established that a laminar zonal flow is generated by the nonlinear self-interaction of the viscous flow in the boundary layer (e.g. Wang 1970; Suess 1971; Busse 2010; Calkins et al. 2010; Noir et al. 2010; Sauret et al. 2010; Chan et al. 2011; Zhang et al. 2011). However, at planetary settings, these laminar flows are expected to be weak and we will focus instead on the generation of turbulent flow.

The generation and characteristics of bulk turbulence driven by mechanical forcing is less well understood but crucial for understanding many planetary processes including tidal dissipation. It is well known that for the Earth, the ocean is primarily responsible for tidal dissipation (e.g. Egbert \& Ray 2003). However, considering other planetary bodies, many previous studies have considered tidal dissipation in the solid planet and neglected any contributions from lower viscosity fluid layers (e.g. Williams \& Boggs 2015). More recent studies have begun to consider the dynamic response of these fluid layers to directly forced resonances from surface gravity waves, planetary Rossby waves where the Coriolis forces of the rotating body provides a restoring force and viscous drag at the fluid-solid interface (e.g. Tyler 2008, 2014; Chen et al. 2014; Matsuyama 2014;
Kamata et al. 2015). In this work, we take a different approach by considering indirectly forced resonances where the Coriolis force alone provides the restoring force and can drive turbulence in the entire fluid layer.

A necessary ingredient for such indirectly forced turbulence is the presence of flows with elliptically deformed streamlines that can then support elliptical instabilities. This instability is a parametric resonance between the elliptically deformed flow and two resonating inertial modes of the system (e.g. Kerswell 2002). The instability was found in elliptically deformed flows driven by tidal and librational forcing and referred to as tide- (libration-) driven elliptical instability, TDEI (LDEI; e.g. Kerswell \& Malkus 1998; Le Bars et al. 2007, 2010; Cébron et al. 2012b). In both TDEI and LDEI, the periodic forcing of an ellipsoidal cavity can generate a triadic resonance between the inviscid elliptically deformed base flow and two inertial modes of the system that can globally destabilize the layer leading to bulk turbulence.

TDEI experiments in a deformable sphere and cylinder have shown, through side-view visualizations, that this triadic resonance between the base flow and two inertial modes can generate either intermittent or saturated turbulence (e.g. Lacaze et al. 2004, 2005; Le Bars et al. 2007, 2010). These works confirmed the instability regime, growth rates and the turbulent transition associated with the TDEI. Recent LDEI studies in rigid ellipsoids have also shown both intermittent and saturated globally turbulent flows (e.g. Cébron et al. 2012b; Noir et al. 2012; Grannan et al. 2014; Favier et al. 2015). The aspects of, and instabilities related to, mechanically forced flows are reviewed in Le Bars et al. (2015).

We perform experiments here measuring, for the first time, the TDEI-driven flow velocities in the equatorial plane of a deformable spherical container. These results are coupled with selected direct numerical simulations (DNS). For these studies, the Ekman number, $E=v /\left(\bar{\Omega}_{\text {spin }} R^{2}\right)$, is defined as the ratio of viscous to Coriolis forces where $v$ is the kinematic viscosity, $\bar{\Omega}_{\text {spin }}$ is the mean rotation rate and $R$ is the r.m.s elliptical boundary radius. For the laboratory experimental and numerical work, the Ekman number is fixed at $E=1.5 \times 10^{-5}$ and $E=5 \times 10^{-5}$, respectively. Such efforts incorporate the more extreme parameters available to experiments with the full flow field available to numerical simulations. 
We extend the previous experimental and numerical studies of libration in a rigid cavity with a fixed deformation at the dimensionless frequency, $f_{\text {lib }}=4$ (e.g. Grannan et al. 2014; Favier et al. 2015). In our studies of TDEI, we fix the dimensionless frequency at $f_{\text {tide }}=4$. This dimensionless frequency is outside the range for directly forced inertial resonances (e.g. Greenspan 1969) and allows us then to focus only on the indirect forcing provided by TDEI mechanism.

By comparing the tidal forcing results from the current work with the previous studies of libration, strong similarities between tidaland libration-driven flows are found, suggesting a generic response of the fluid layer independent of the specific forcing. In Section 2, the mathematical framework is developed for tide- and librationdriven flows, the experimental method is described in Section 3 , and the numerical method is outlined in Section 4. The results for tide-driven flows and their comparison with libration-driven flows are provided in Section 5. Finally, our conclusions are summarized in Section 6.

\section{MATHEMATICAL BACKGROUND}

In our tidal forcing scenario, we consider a homogenous, incompressible, Newtonian fluid that is enclosed in an ellipsoidal container. The boundary of this ellipsoid is specified by, $x^{2} / a^{2}+y^{2} / b^{2}+z^{2} / c^{2}=1$, set in a Cartesian coordinate system affixed to the ellipsoid with the long axis, $\hat{\mathbf{x}}$, of length $a$. The short equatorial axis with length $b$ is along $\hat{\mathbf{y}}$ while $\hat{\mathbf{z}}$ is along the axis of rotation with length $c$. Non-dimensionalizing the ellipsoid equation using the r.m.s. fluid layer length scale, $R=\sqrt{\left(a^{2}+b^{2}\right) / 2}$, yields:

$\frac{x^{2}}{1+\beta}+\frac{y^{2}}{1-\beta}+\frac{z^{2}}{c^{* 2}}=1$.

The equatorial ellipticity of the cavity is defined as $\beta=\left(a^{2}-b^{2}\right) /\left(a^{2}+b^{2}\right)$, while the axial deformation is $c^{*}=c / R$.

From a numerical perspective, it is advantageous to work in the reference frame fixed to the elliptical distortion, termed the orbital frame rotating at $\Omega_{\text {orb }}$. The generalized equations of motion:

$$
\frac{\partial \mathbf{u}}{\partial t}+\mathbf{u} \cdot \nabla \mathbf{u}+2 \gamma(t) \hat{\mathbf{z}} \times \mathbf{u}=-\nabla \Pi+E \nabla^{2} \mathbf{u}-\frac{\partial \gamma(t)}{\partial t} \hat{\mathbf{z}} \times \mathbf{x},
$$

$$
\nabla \cdot \mathbf{u}=0
$$

are non-dimensionalized using $R$ as the length scale and the mean spin rate of the fluid, $\bar{\Omega}_{\text {spin }}^{-1}$, as the time scale. In (2), the first two terms on the left-hand side are the inertial terms, and the third term is the Coriolis acceleration. Here,

$\gamma_{\text {tide }}=\frac{\Omega_{\text {orb }}}{\bar{\Omega}_{\text {spin }}}$,

is the ratio of the elliptical distortion rotation rate to the mean spin rate of the fluid. The forcing frequency felt by a fluid parcel, $\omega_{\text {tide }}$, due to the elliptical distortion is related to the both rotation rates by $\omega_{\text {tide }}=2\left(\bar{\Omega}_{\text {spin }}-\Omega_{\text {orb }}\right)$. The dimensionless forcing frequency due to the elliptical distortion is then $f_{\text {tide }}=\omega_{\text {tide }} / \bar{\Omega}_{\text {spin }}=2\left(1-\gamma_{\text {tide }}\right)$. On the right-hand side of (2), $\Pi$ is the modified pressure term where the centrifugal acceleration, $-\gamma(t) \hat{\mathbf{z}} \times \gamma(t) \hat{\mathbf{z}} \times \mathbf{x}=\nabla \gamma^{2}(t)\left(x^{2}+\right.$ $\left.y^{2}\right) / 2$, is absorbed into the pressure gradient. The next term on the right is the viscous diffusion term where $E=v /\left(\bar{\Omega}_{\text {spin }} R^{2}\right)$ is the Ekman number defining the ratio of the viscous forces to Coriolis forces where $v$ is the kinematic viscosity.
The final term on the right-hand side is the Poincaré acceleration associated with the time-dependent elliptical distortion rotation rate. This term is zero for the current tidal forcing studies since $\gamma_{\text {tide }}$ is constant. For tidal forcing, an impermeable, no-slip boundary condition is implemented with a horizontal tangential velocity given by

$\mathbf{u}_{\text {b.c. }}=\left(1-\gamma_{\text {tide }}\right) \sqrt{\frac{a^{\prime 2}+b^{\prime 2}}{2}} \boldsymbol{\tau}$,

where $\boldsymbol{\tau}$ is the normalized tangent vector and $\left[a^{\prime}, b^{\prime}\right]=$ $[a, b] \sqrt{1-z^{2} / c^{2}}$. More details are provided in Appendix A.

In the studies of longitudinal libration, the reference frame is fixed to the elliptical distortion such that the equations of motion in (2) and (3) are still used. However, the ratio of the elliptical distortion rotation rate to the mean spin rate of the fluid now takes the form:

$\gamma_{\text {lib }}(t)=\frac{\Omega_{\text {spin }}(t)}{\bar{\Omega}_{\text {spin }}}=\left(1+\epsilon_{\text {lib }} \sin \left(f_{\text {lib }} t\right)\right)$,

where $\epsilon_{\text {lib }}=f_{\text {lib }} \Delta \phi$ is the dimensionless amplitude of librational oscillation where the dimensionless frequency ratio, $f_{\text {lib }}=\omega_{\text {lib }} / \bar{\Omega}_{\text {spin }}$, is the ratio of the elliptical distortion frequency to the mean rotation rate and $\Delta \phi$ is the librational amplitude in radians (e.g. Cébron et al. 2012b; Noir et al. 2012; Sauret 2012; Favier et al. 2015). Because of the time-dependence in (6), the Poincare term in (2) is now non-zero. Finally, an impermeable, no-slip condition is implemented with $\mathbf{u}_{\text {b.c. }}=0$.

An inviscid solution of (2), generalized for tidal or librational forcing in the orbital reference frame, is given in Cébron et al. (2012a) as

$\mathbf{U}_{B}=\left(1-\gamma_{\text {tide, lib }}\right)\left(\begin{array}{ccc}0 & -(1+\beta) & 0 \\ 1-\beta & 0 & 0 \\ 0 & 0 & 0\end{array}\right) \mathbf{x}$

where $\mathbf{x}=(x, y, z)$ is a general position vector and $\left(1-\gamma_{\text {tide,lib }}\right)$ is the forcing amplitude. The maximum amplitude of this forcing is defined as $\epsilon_{\text {tide,lib }}=\left(1-\gamma_{\text {tide,lib }}\right)_{\max }$. The purely horizontal base flow in (7) satisfies a non-penetration condition at the boundary and follows the ellipsoidal shape of the container characterized by an azimuthal wavenumber in cylindrical coordinates, $m_{\text {tide, lib }}=2$, and forced at the dimensionless frequency $f_{\text {tide, lib }}$ contained in the forcing amplitude $\left(1-\gamma_{\text {tide, lib }}\right)$. The total kinetic energy associated with this flow in the orbital frame is

$E_{B}=\frac{4 \pi}{15}\left(1-\gamma_{\text {tide, lib }}\right)^{2}\left(1-\beta^{2}\right)^{3 / 2} c^{*}$.

It is experimentally advantageous to work in the spin frame fixed at $\bar{\Omega}_{\text {spin }}$ and, as such, the base flow is transformed into the spin frame where

$\mathbf{U}_{B_{\mathrm{rot}}}=\left(1-\gamma_{\mathrm{tide}, \mathrm{lib}}\right) \beta\left(\begin{array}{ccc}-\sin (2 \theta(t)) & -\cos (2 \theta(t)) & 0 \\ -\cos (2 \theta(t)) & \sin (2 \theta(t)) & 0 \\ 0 & 0 & 0\end{array}\right) \mathbf{x}_{\mathrm{rot}}$.

Here $\mathbf{x}_{\text {rot }}=(X, Y, Z)$ is a general position vector in the spin frame and $\theta(t)=\int_{0}^{t}\left(1-\gamma_{\text {tide, lib }}\left(t^{\prime}\right)\right) \mathrm{d} t^{\prime}$ is the total angle between the axes of the spin frame and the orbital frame. The total kinetic energy associated with this flow in the spin frame is

$E_{B_{\mathrm{rot}}}=\frac{4 \pi}{15}\left(1-\gamma_{\mathrm{tide}, \mathrm{lib}}\right)^{2} \beta^{2}\left(1-\beta^{4}\right) c^{*}$. 
Table 1. Comparison of tidal and librational forcing parameters. The definitions for equatorial ellipticity, $\beta$, mean rotation rate, $\bar{\Omega}_{\text {spin }}$ and the Ekman number, $E$ are shared by both types. The differences arise in how the mechanical forcing is implemented at the boundary. In the current work, $f_{\text {tide,lib }}=4$ is fixed allowing for a comparison between both mechanisms.

\begin{tabular}{|c|c|c|c|}
\hline Parameter & Definition & Tides & Libration \\
\hline Equatorial ellipticity & $\beta$ & $\frac{a^{2}-b^{2}}{a^{2}+b^{2}}$ & $\frac{a^{2}-b^{2}}{a^{2}+b^{2}}$ \\
\hline Mean rotation rate & & $\bar{\Omega}_{\text {spin }}$ & $\bar{\Omega}_{\text {spin }}$ \\
\hline Elliptical distortion rotation rate & & $\Omega_{\text {orb }}$ & $\Omega_{\text {spin }}(t)=\bar{\Omega}_{\text {spin }}+\omega_{\text {lib }} \Delta \phi \sin \left(\frac{\omega_{\text {lib }}}{\bar{\Omega}_{\text {spin }}} t\right)$ \\
\hline Elliptical distortion frequency & & $\omega_{\text {tide }}=2\left(\bar{\Omega}_{\text {spin }}-\Omega_{\text {orb }}\right)$ & $\omega_{\mathrm{lib}}$ \\
\hline Rotation rate ratio & $\gamma_{\text {tide, lib }}=\frac{\text { Elliptical dist. rotation rate }}{\text { Mean rotation rate }}$ & $\frac{\Omega_{\text {orb }}}{\overline{\Omega_{\text {spin }}}}$ & $1+f_{\mathrm{lib}} \Delta \phi \sin \left(f_{\mathrm{lib}} t\right)$ \\
\hline $\begin{array}{l}\text { Forcing amplitude } \\
\text { Inviscid growth rate }\end{array}$ & $\begin{array}{l}\epsilon_{\text {tide,lib }}=\left(1-\gamma_{\text {tide, lib }}\right)_{\max } \\
\sigma_{\text {inv }}^{\text {tide lib }}\end{array}$ & $\begin{array}{l}f_{\text {tide }} / 2 \\
\frac{\epsilon_{\text {tide }} \beta}{64}\left(4+f_{\text {tide }}\right)^{2}\end{array}$ & $\begin{array}{l}f_{\mathrm{lib}} \Delta \phi \\
\frac{\epsilon_{\mathrm{lib} \beta} \beta}{16}\left(16+f_{\mathrm{lib}}\right)^{2}\end{array}$ \\
\hline
\end{tabular}

To satisfy the no-slip boundary conditions, viscous corrections in the Ekman boundary layer generate a flow linear in $\beta$ that connects the base flow with the no-slip boundary. The nonlinear self-interaction of this $m_{\text {tide,lib }}=2$ flow generates flows of azimuthal wavenumbers $m=4$ and $m=0$, the latter of which is associated with an axisymmetric, steady zonal flow that scales with $\beta^{2}$ in the boundary layer and is independent of the Ekman number (e.g. Sauret 2012).

Perturbations in rotating fluids can generate inertial waves whose restoring force is the Coriolis force and whose dimensionless frequency is given by the dispersion relation, $\lambda=\omega_{\text {tide, lib }} / \bar{\Omega}_{\text {spin }}=$ $\pm 2 \cos \theta$, where $\theta$ is the angle between the wave-vector and the axis of rotation. In closed cavities, reflections and constructive interference support inertial modes with the same frequency range, namely $-2 \lesssim \lambda \lesssim 2$ (e.g. Greenspan 1969; Vantieghem 2014). Mechanical forcing can excite these inertial modes, in addition to generating the aforementioned elliptically deformed base flow (7). An elliptical instability, (EI) can be generated when two inertial mode frequencies and azimuthal wavenumbers are related to those of the base flow given in Kerswell (2002) by:

$\left|\lambda_{1}-\lambda_{2}\right|=f_{\text {tide, lib }}$,

$\left|m_{1}-m_{2}\right|=m_{\text {tide, lib }}=2$.

Using the inertial mode frequency range, the maximum frequency for exciting EI is $f_{\text {tide, lib }} \lesssim 4$. Uniquely for tides, since the tidal forcing frequency is given by $f_{\text {tide }}=2\left(1-\gamma_{\text {tide }}\right)$, this unstable range can be written as $-1 \leq \gamma_{\text {tide }} \leq 3$ (e.g. Le Bars et al. 2010). In contrast, for libration, $\gamma_{\text {lib }}$, in (6) can take any value to drive elliptical instability as long as the elliptical distortion frequency $\omega_{\text {lib }}$ is no greater than four times the mean rotation rate (i.e. $\omega_{\text {lib }} / \bar{\Omega}_{\text {spin }}=f_{\text {lib }} \lesssim 4$ ).

To calculate the inviscid growth rates, local stability analyses were performed based on the Wentzel-Kramers-Brillouin method in Le Dizes (2000). There it was shown, in the limit of $\epsilon_{\text {tide,lib, }}$ $\beta \ll 1$, that the inviscid growth rates for TDEI and LDEI are given respectively as:

$\sigma_{\text {inv }}^{\text {tide }}=\frac{\epsilon_{\text {tide }} \beta}{64}\left(4+f_{\text {tide }}\right)^{2}$,

$\sigma_{\mathrm{inv}}^{\mathrm{lib}}=\frac{\epsilon_{\mathrm{lib}} \beta}{16}\left(16+f_{\mathrm{lib}}\right)^{2}$.

The similarity of the growth rates in (13) and (14) suggest that the response of a localized fluid parcel away from the elliptical boundary is independent of the forcing mechanism. The general form of the equation including viscous effects and the proximity of the forcing frequency to some resonant frequency, $f_{\text {res }}$, is given from Cébron et al. (2012a) as:

$\sigma=\sqrt{\sigma_{\text {inv }}^{2}-\left(f_{\text {res }}-f\right)^{2}}-K \sqrt{E}$.

The second term on the right-hand side of (15) is due to viscous dissipation in the Ekman boundary where $K$ is a constant typically $[1-10]$. Table 1 provides a comparison of tidal and librational formulae.

\section{EXPERIMENTAL METHOD}

The experimental setup, shown in Fig. 2(a) is adapted from the same apparatus used previously in Le Bars et al. (2010), Morize et al. (2010), Sauret et al. (2010, 2014). A hollow sphere is enclosed in a cast cylinder of deformable semi-transparent silicone gel. Tidal forcing is replicated using two motors. The first motor rotates the sphere about the $\hat{\mathbf{z}}$ axis at a constant counter-clockwise rate, $\bar{\Omega}_{\text {spin }} / 2 \pi=1.05 \mathrm{~Hz}$. The second motor generates the tidal deformation by rotating two symmetric axial rollers pressed radially into the silicone at a fixed clockwise rate, $\Omega_{\text {orb }} / 2 \pi=1.03 \mathrm{~Hz}$. The rotation rate ratio of the two motors is fixed at $\gamma_{\text {tide }}=\Omega_{\text {orb }} / \bar{\Omega}_{\text {spin }} \simeq-1$, indicating that the rates are nearly equal and moving in opposite directions. The amplitude of the equatorial ellipticity in the tidal forcing, the amount by which the orbiting rollers are pressed radially inward, is given by, $\beta=\left(a^{2}-b^{2}\right) /\left(a^{2}+b^{2}\right) \in[0.01-0.09]$. For completeness, the axial deformation $c^{*}=c / R \sim 1$, where $R=\sqrt{\left(a^{2}+b^{2}\right) / 2} \sim 10 \mathrm{~cm}$ for all cases. Finally, the Ekman number defining the ratio of viscous forces to the Coriolis forces is fixed at $E=1.5 \times 10^{-5}$ using water, for which $v \cong 10^{-6} \mathrm{~m}^{2} \mathrm{~s}^{-1}$.

For comparison, the libration setup used in Grannan et al. (2014) is included in Fig. 2(b). In brief, two motors generate a constant background rotation, $\bar{\Omega}_{\text {spin }}$, and superimposed sinusoidal oscillations at a fixed Ekman number, $E=v /\left(\bar{\Omega}_{\text {spin }} R^{2}\right)=2.7 \times 10^{-5}$. Details of the experimental method are found in Grannan et al. (2014) and Table 2 provides a comparison of laboratory experimental tidal and librational forcing parameters.

To make quantitative measurements in the tidal forcing laboratory experiments, a particle image velocimetry (PIV) technique is employed in the $\bar{\Omega}_{\text {spin }}$ reference frame. Nearly spherical, $100 \mu \mathrm{m}$ diameter Optimage particles of density $\left(1 \pm 0.02 \mathrm{~g} \mathrm{~cm}^{-3}\right)$ are added to the water. Two oppositely faced laser light sheets are fixed in the laboratory frame several millimetres above the equatorial plane 

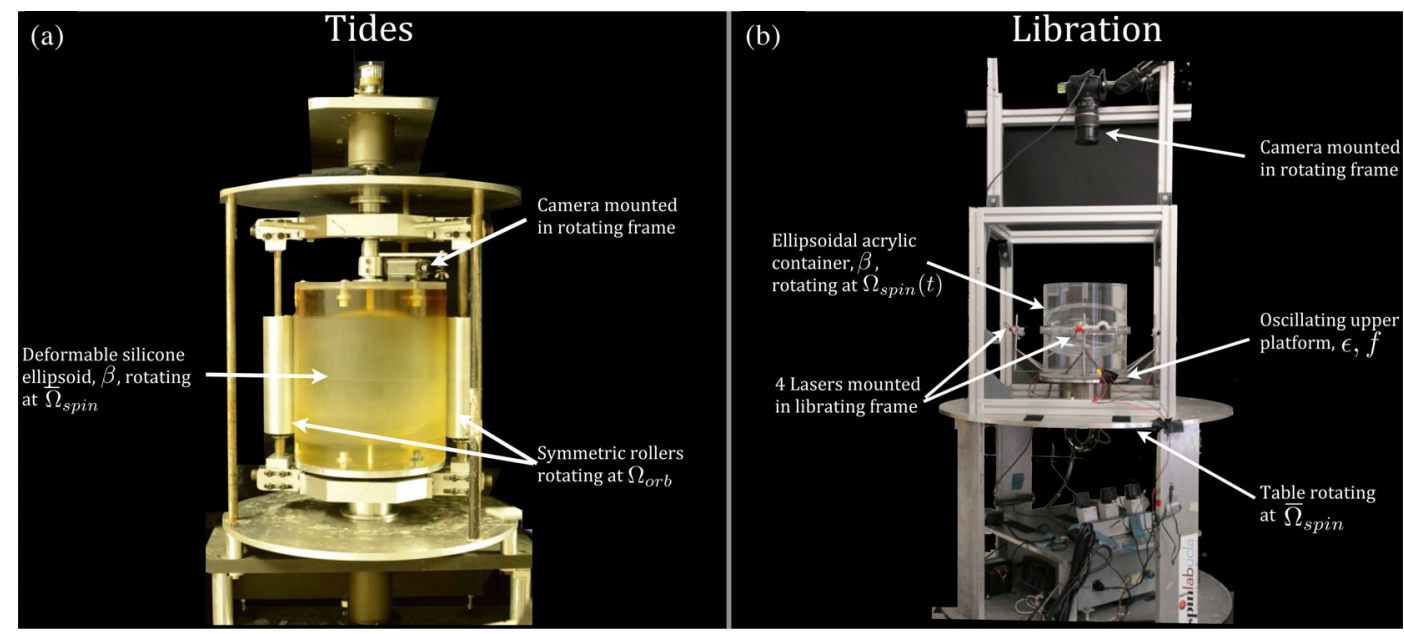

Figure 2. Comparison of tide and libration experimental setups. (a) The tidal deformation experiment where the camera is fixed to a deformable container with equatorial ellipticity, $\beta$, rotating at $\bar{\Omega}_{\text {spin. }}$. The two symmetric rollers rotate independently at $\Omega_{\text {orb }}$. (b) The libration experimental setup used in Grannan et al. (2014). A rigid ellipsoid with equatorial ellipticity, $\beta$, rotates at a constant spin rate and oscillates using two motors $\operatorname{such}$ that $\Omega_{\text {spin }}(t)=\bar{\Omega}_{\text {spin }}\left(1+\epsilon_{\text {lib }} \sin \left(f_{\text {lib }} t\right)\right)$. Image from (b) adapted from Grannan et al. (2014).

Table 2. Comparison of laboratory experimental tidal and librational forcing parameters. The definitions for equatorial ellipticity, $\beta$, mean rotation rate, $\bar{\Omega}_{\text {spin }}$ and the Ekman number, $E$ are shared by both types. The differences arise in how the mechanical forcing is implemented at the boundary. However, in the current work, $f_{\text {tide,lib }} \simeq 4$ is fixed allowing for a comparison between both mechanisms.

\begin{tabular}{lccc}
\hline Parameter & Definition & Tides & Libration \\
\hline$a$ & Long equatorial axis $(\mathrm{cm})$ & $10.05-10.8 \mathrm{~cm}$ & $12.7 \mathrm{~cm}$ \\
$b$ & Short equatorial axis $(\mathrm{cm})$ & $9.95-9.2 \mathrm{~cm}$ & $8.9 \mathrm{~cm}$ \\
$c$ & Short rotational axis $(\mathrm{cm})$ & $10 \mathrm{~cm}$ & $8.9 \mathrm{~cm}$ \\
$\beta$ & $\frac{a^{2}-b^{2}}{a^{2}+b^{2}}$ & $0.01-0.16$ & 0.34 \\
$R$ & $\sqrt{\frac{a^{2}+b^{2}}{2}}$ & $10 \mathrm{~cm}$ & $10.97 \mathrm{~cm}$ \\
$v$ & Kinematic viscosity & $10^{-6} \mathrm{~m}^{2} \mathrm{~s}^{-1}$ & $10^{-6} \mathrm{~m}^{2} \mathrm{~s}^{-1}$ \\
$\bar{\Omega}_{\text {spin }} / 2 \pi$ & Mean rotation rate & $1.05 \mathrm{~Hz}$ & $0.5 \mathrm{~Hz}$ \\
$\Omega_{\text {orbit }} / 2 \pi$ & Elliptical distortion rotation rate & $-1.03 \mathrm{~Hz}$ & $0.5(1+0.8 \sin (4 \pi t)) \mathrm{Hz}$ \\
$\omega_{\text {tide,lib }} / 2 \pi$ & Elliptical distortion frequency & $4.16 \mathrm{~Hz}$ & $2.0 \mathrm{~Hz}$ \\
$c^{*}$ & $\frac{c}{R}$ & 1 & 0.81 \\
$f_{\text {tide, }, \text { ib }}$ & $\omega_{\text {tide, lib }} / \bar{\Omega}_{\text {spin }}$ & 3.96 & 4.0 \\
$\gamma_{\text {tide,lib }}$ & Mean rotation rate & -0.98 & $1+0.8 \sin (4 \pi t)$ \\
$\epsilon_{\text {tide, lib }}$ & $\left(1-\gamma_{\text {tide,lib }}\right)_{\text {max }}$ & 1.98 & 0.8 \\
$E$ & $\bar{\Omega}_{\text {spin } R^{2}}$ & $1.5 \times 10^{-5}$ & $2.7 \times 10^{-5}$ \\
\hline
\end{tabular}

due to the presence of the joining seam of the silicone hemispheres. Mirrors are also implemented in the laboratory frame to create a more uniform laser light sheet. A GoPro Hero3+ camera is fixed in the spin frame, in order to acquire $1920 \times 1280$ resolution movies of the horizontal flow field at 60 frames per second. These movies are made only after solid body rotation has been reached; they are initiated at the start of tidal forcing and the recordings are typically 10 minutes in duration. The camera is controlled wirelessly using a GoPro smartphone application.

All movies are separated into their constituent frames and preprocessed to remove the Go-Pro's fish-eye distortion and to adjust the brightness and contrast. All adjusted images are passed through Meunier \& Lewecke's (2003) open source PIV software, DPIVSoft2010, that has been successfully employed in previous studies (e.g. Morize et al. 2010; Sauret et al. 2010; Grannan et al. 2014; Sauret et al. 2014). The velocity field for an entire equatorial plane is resolved spatially into a $40 \times 60$ grid with a typical resolution of $3 \mathrm{~mm}$ and the temporal resolution of the PIV is $59 \mathrm{~Hz}$. The practical dimensional and non-dimensional temporal frequency limit for our setup is $\sim 8 \mathrm{~Hz}$ and $f \sim 8$, respectively. For higher frequencies above $f>8$ where the power spectra falls below $\mathcal{O}\left(10^{-5}\right)$, the noise is on the order of the signal. This frequency cut-off is shown in Fig. 8 and is discussed in Section 5.3.

All velocity measurements presented below are nondimensionalized using the radius, $R$, and spin period $\bar{\Omega}_{\text {spin }}^{-1}$. Thus, the dimensional velocity is found by multiplying the dimensionless velocity by $\bar{\Omega}_{\text {spin }} R$. The dimensional energy density is found by multiplying the dimensionless energy by $\rho \bar{\Omega}_{\text {spin }}^{2} R^{2}$.

\section{NUMERICAL METHOD}

Numerical simulations were performed with the massively parallel spectral-element code Nek5000 (http://nek5000.mcs.anl.gov) developed by Paul Fischer and collaborators (see Fischer et al. 2007, and references within). Nek5000 solves the incompressible NavierStokes equations via a Legendre polynomial based spectral element method which combines the geometrical flexibility of finite ele- 
ment methods with the accuracy of spectral methods. It is therefore particularly well adapted to our problem involving turbulent flows in complex non-axisymmetric geometries and has been used previously in the context of tidally forced spheres and librationally forced elliptical flows (e.g. Favier et al. 2014a, 2015; Barker et al. 2016).

\section{RESULTS}

The laboratory experimental cases investigated in this work are denoted by $L-$ while the numerical cases are denoted by $N-$. The parameters for all the experimental and numerical cases studied are listed in Tables B1 and B2 respectively of Appendix B.

\subsection{General properties}

Fig. 3 shows, for the first time, the strong similarities found between the TDEI excited in the experiments and numerics of the current work with the LDEI found in the previous works of Grannan et al. (2014); Favier et al. (2015). Kalliroscope visualizations are illuminated by an axial laser light sheet in order to take high resolution movies of the experiments. The tidal forcing images in Fig. 3(a) are taken from case $L 5,\left(\beta=0.06, E=1.5 \times 10^{-5}\right)$ in the laboratory frame while librational forcing images of Fig. 3(c) from case $V,\left(\beta=0.34, E=2.7 \times 10^{-5}\right)$ in Grannan et al. (2014) are taken in the rotating frame. Snapshots of the numerical simulations in Figs 3(b) and (d) show the axial velocities from the TDEI in case $N 4,\left(\beta=0.09, E=5 \times 10^{-5}\right)$ and the LDEI from case $A 6$, ( $\left.\beta=0.34, E=10^{-4}\right)$ of Favier et al. (2015) in the ellipsoidal frame.

Moving from left to right through the three time steps of Fig. 3, after solid body rotation has been reached, the forcing commences with no visible flow in the bulk. Since the base flow is purely horizontal, the numerical simulations in Figs 3(b) and (d) show that axial velocity is non-zero only in the thin boundary layer. After some time, for forcing above a critical value such that the growth rate in (15) is positive, the excited inertial modes participating in the instability grow and become visible. Since $f_{\text {tide,lib }}=4$ is at the limit of elliptical instability, the coupled inertial modes are necessarily $\left|\lambda_{1,2}\right|=f_{\text {tide, lib }} / 2 \approx 2$ by (11), and the wave crests are perpendicular to the rotation axis as indicated by the layered structures. It is noted that inviscid inertial modes with $|\lambda|=2$ exactly do not exist but may be just below 2 due to viscous effects and imperfect resonances. On the right of Fig. 3, the growth of the inertial modes leads to a wave breaking event that transitions to sustained bulk filling small-scale turbulence that does not return to the laminar base state found at early times.

Side-view visualizations reveal that as the strength of tidal deformation is varied between $\beta \in[0.01-0.16]$, the transition to bulk turbulence, shown in Fig. 3, is seen in all cases except for $\beta=0.01$ which remains laminar for all times. Using this critical value for tidal deformation, $f_{\text {res }}=4$, and setting the growth rate in eq. (15) equal to zero, the dissipation constant is then $K=3$ and 5 for DNS and experiments, respectively. These $K$ values are within the typical range for $K=[1-10]$ also found in previous studies (e.g. Cébron et al. 2012b; Noir et al. 2012).

The onset of turbulence is quantified using the averaged axial kinetic energy defined as

$E_{z}=\frac{1}{2 V} \int_{V} u_{z}^{2} \mathrm{dV}$,

where $V=4 / 3 \pi a b c$ is the ellipsoidal volume and $u_{z}$ is the axial velocity component. This diagnostic is a natural choice since the tide-induced base flow of (7) and (9) are purely horizontal and any increase in the axial energy indicates the excitement of the TDEI.

Additionally, these flow quantities vary in time due to the oscillating base flow and may be sufficiently smoothed by averaging over one spin period defined as

$\bar{A}(t)=\frac{1}{\tau_{\text {spin }}} \int_{t}^{t+\tau_{\text {spin }}} A\left(t^{\prime}\right) \mathrm{d} t^{\prime}$,

where $\tau_{\text {spin }}=2 \pi / \bar{\Omega}_{\text {spin }}$.

The axial kinetic energy in (16) is not measurable in the experimental PIV which is limited to only the horizontal energy. Since the experimental data is inherently more noisy, the horizontal flows for experiments and DNS are computed by subtracting out the base flow in (7) and (9), respectively. After the base flow is removed from the experimental data, the horizontal energy in the bulk is integrated, in the rotating frame, over an equatorial surface area with a maximum radius $0.5 R$ where $R=\sqrt{a^{2}+b^{2}} / 2$ :

$E_{H}^{S}=\frac{1}{2 S} \int_{S}\left(\mathbf{u}_{H_{\mathrm{rot}}}-\mathbf{U}_{B_{\mathrm{rot}}}\right)^{2} \mathrm{dS}$.

In comparison, the volume averaged horizontal energy of the DNS is computed in the ellipsoidal frame by

$E_{H}^{V}=\frac{1}{2 V} \int_{V}\left(\mathbf{u}_{H}-\mathbf{U}_{B}\right)^{2} \mathrm{dV}$.

Fig. 4(a) shows the transition from laminar to turbulent flow as the equatorial ellipticity, $\beta$, is increased by plotting the unaveraged horizontal energy from (18) in grey and the time average in colour as a function of the number of spin periods $\tau_{\text {spin }}$ for the experiments. The laminar case, $\beta=0.01$, (blue line) shows no transition after the base flow is established at early times.

For $\beta \in[0.03-0.16]$, after the base flow is established, the energy grows exponentially followed by an overshoot and then oscillations around a saturated phase. This is corroborated in Fig. 4(b) where an analysis of the volume averaged horizontal energy computed in the ellipsoidal frame of the DNS reveals the same transitions. Once the transition occurs we find no evidence for relaminarization wherein the flow returns to the laminar base state found at early times. For all cases, the energy of the unstable flow is quite small, $\mathcal{O}\left(10^{-3}-10^{-2}\right)$, indicating that although the instability conveys just a small portion of the available kinetic energy of the system, first order changes are generated in the flow.

To verify the characteristics of the EI mechanism, we focus on the transitions in the axially averaged energy of the DNS in Figs 4(c) and (d). The growth rates for different $\beta$ are predicted using the theoretical growth rate given by (15) for fixed $f_{\text {tide }}=4, E=5 \times 10^{-5}$ and $K=3$ plotted using solid black lines of the form, $e^{2 \sigma t}$, in Fig. 4(c). The slope of the exponential phase is well-matched with the theoretical prediction. Note that the same analysis of the exponential growth rate of the horizontal energy shown in Figs 4(a) and (b) is also in general agreement.

Because the base flow in (7) is purely horizontal, the non-zero axial energy for early times in Fig. 4(c) is associated with viscous corrections in the boundary proportional to $\beta^{2}$. Thus, normalizing the axial energy by $\beta^{2}$ collapses all the energy profiles to a constant value as shown in Fig. 4(d) at early times. Unexpectedly, at later times after the exponential growth of the flow energy, the amplitudes of the saturated turbulent energy also collapse to oscillations around a constant value as shown by the black horizontal line and indicate that the saturation energy also scales with $\beta^{2}$. By extension, the saturation amplitude for the turbulent velocity, $U_{\mathrm{TDEI}}$, is then

$U_{\mathrm{TDEI}} \sim \beta$. 


\section{Tides}

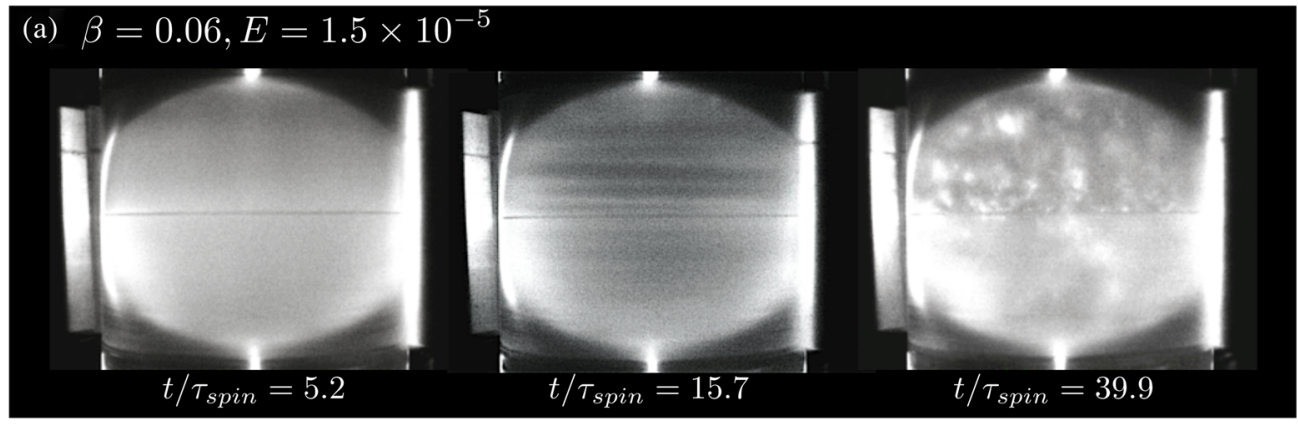

(b) $\beta=0.09, E=5 \times 10^{-5}$
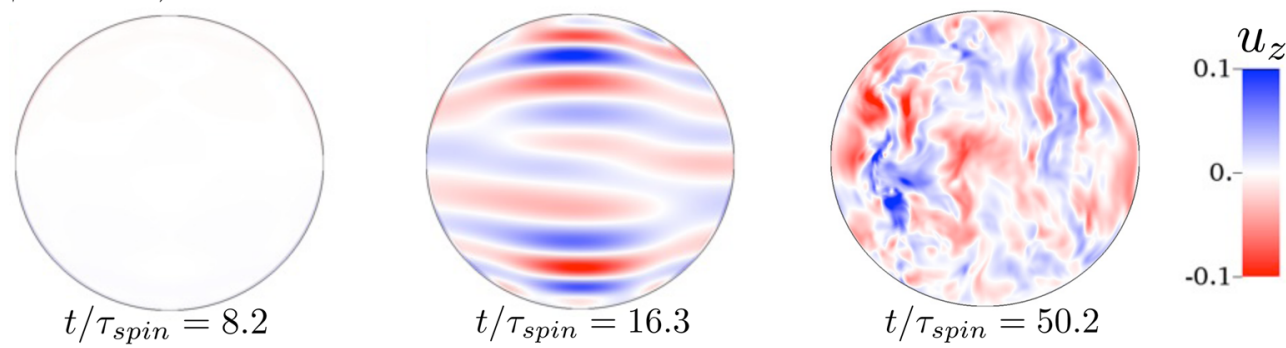

$-0.1$

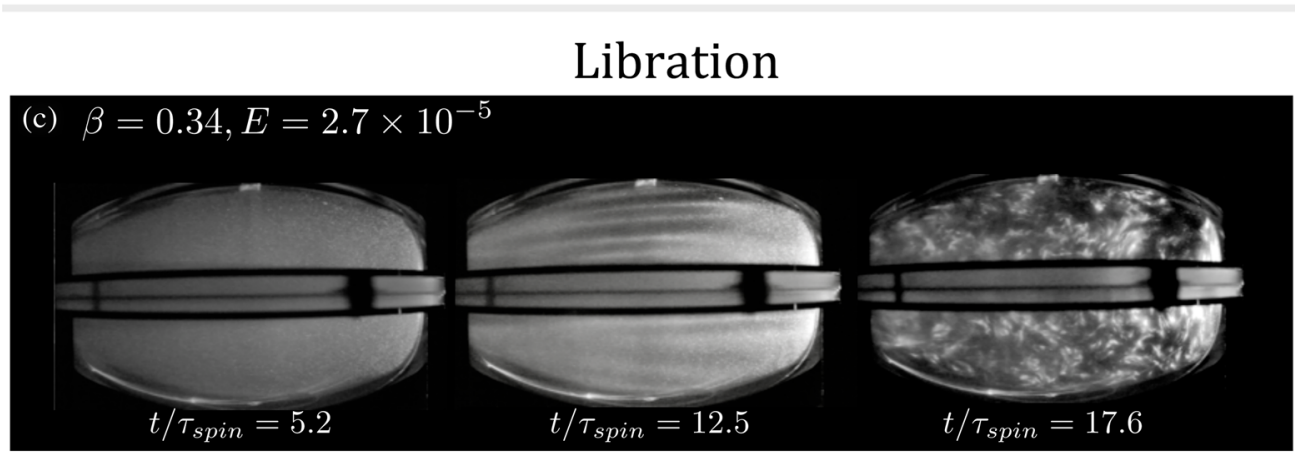

(d) $\beta=0.34, E=10^{-4}$
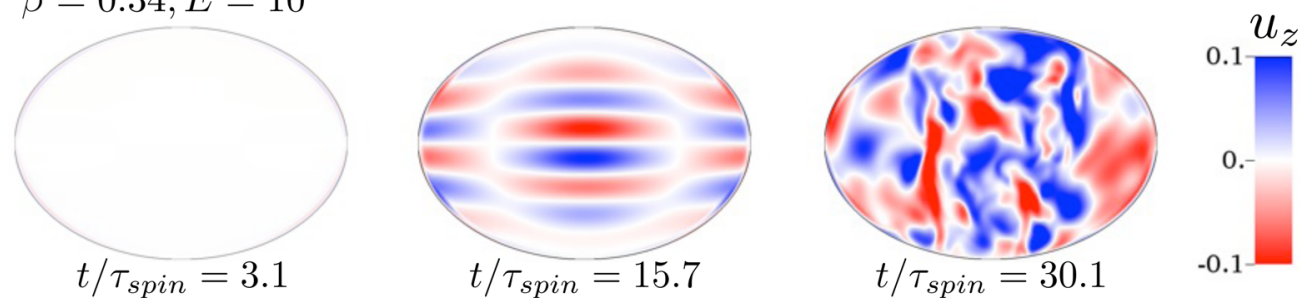

Figure 3. Comparison of side-view visualizations showing the onset of the $\mathrm{EI}$ at $f_{\text {tide,lib }}=4$. For all cases, from left to right, first the base flow is established in the bulk and axial flow occurs in the boundary layer, followed by the stacked structures indicating the growing inertial modes, and finally the breakdown to sustained bulk turbulence. For experiments, Kalliroscope visualizations of the meridional flows illuminated by axial laser light sheets are shown while the axial velocity is shown for the numerical simulations. (a) Tidally forced flow case $L 5$ where $\beta=0.06$ and $E=1.5 \times 10^{-5}$ as seen in the laboratory frame. (b) Excitation of TDEI from case $N 4$ where $\beta=0.09$ and $E=5 \times 10^{-5}$. (c) LDEI experimental case from $V$ in Grannan et al. (2014) with frequency $f_{\text {lib }}=4$, equatorial ellipticity $\beta=0.34$ and Ekman number $E=2.7 \times 10^{-5}$ seen in the rotating frame. (d) Numerical simulation of case $A 6$ in Favier et al. (2015) showing the excitation of the LDEI where $\beta=0.34$ and $E=10^{-4}$.

Note that since $\beta \ll 1$ in the current work and in planetary settings, this novel scaling for the turbulent saturation velocity is larger than the amplitude associated with laminar zonal flows that scale with $\beta^{2}$. Note that a collapse of the saturation in the horizontal flows in Figs 4(a) and (b) is also found, but is not shown for brevity.

In Fig. 4 we also note a general increase in the frequency of the oscillations as $\beta$ increases during the saturated flow phase. This increase is expected because as the saturation velocity scales with $\beta$, the oscillations after the exponential growth phase correspond to a typical oscillation frequency that scales as $U_{\mathrm{TDEI}} / \ell \sim \mathcal{O}(\beta)$ for a fixed length scale $\ell$. The relation between these oscillations and the participating inertial modes requires a more complete analysis of the long term evolution characteristics of the flow and is currently being performed using numerical simulations in a local Cartesian geometry (i.e. Barker \& Lithwick 2013). 
(a) Laboratory

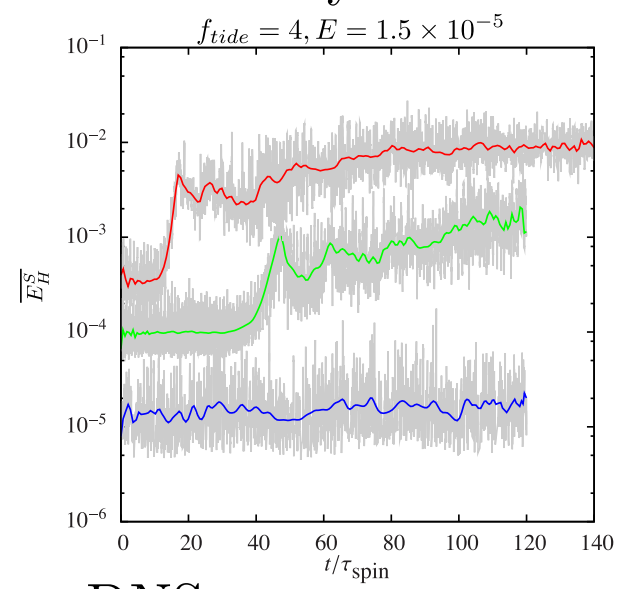

(c) DNS

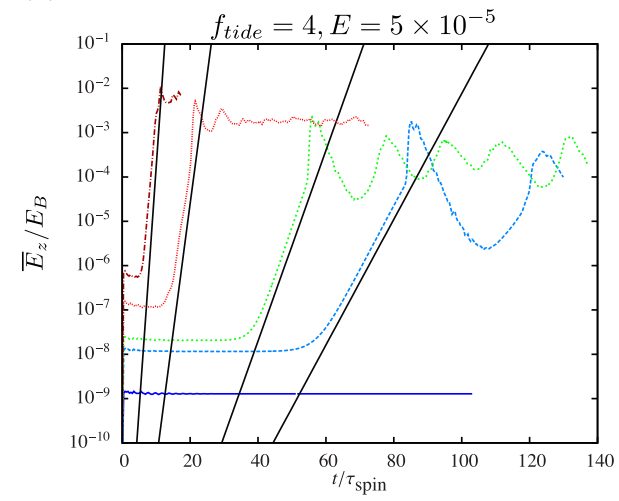

(b) DNS

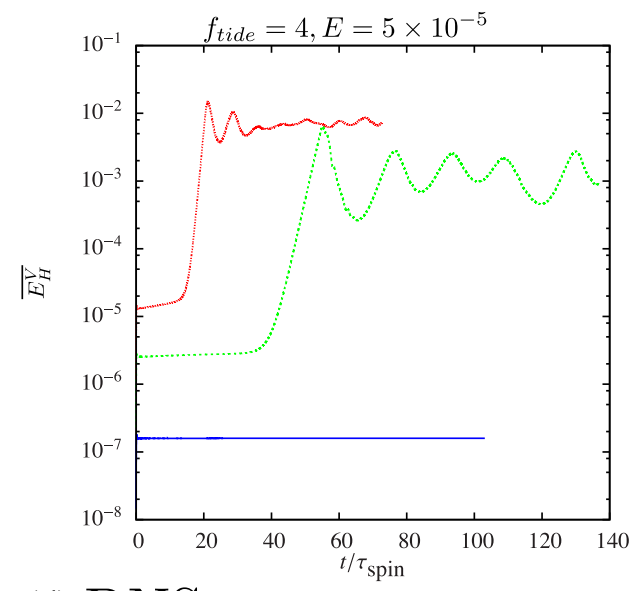

(d) DNS

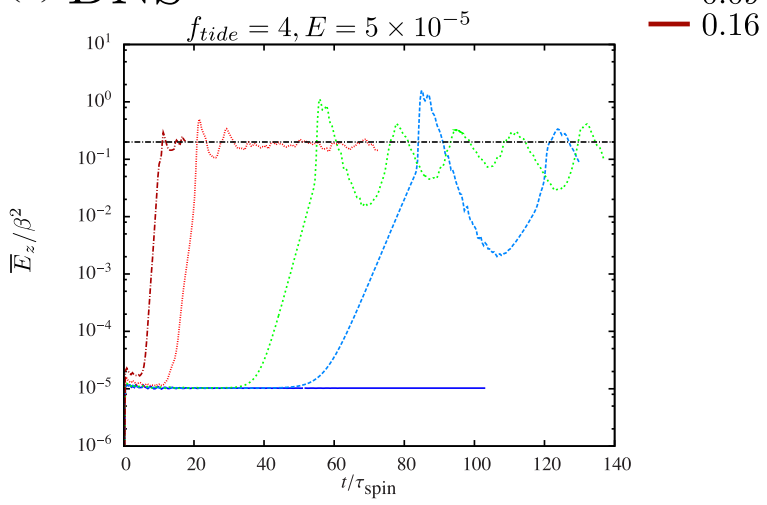

Figure 4. Tidally driven laboratory experiments and numerical simulations. (a) Laboratory measurements of the instantaneous surface averaged horizontal energy in the rotating frame with the base flow removed, $E_{H}^{S}$, as defined in (18) and shown in grey. The coloured curves correspond to the averages over a spin period following (17). The cases shown are $L 1, \beta=0.01, L 3, \beta=0.04$ and $L 8, \beta=0.09$. (b) For numerical simulations, the volume averaged horizontal energy in the ellipsoidal frame with the base flow removed, $E_{H}^{V}$, as defined in (19). The cases shown are $N 1, \beta=0.01, N 3, \beta=0.04$ and $N 4, \beta=0.09$. (c) Time evolution of field-averaged axial kinetic energy $\bar{E}_{z}$ normalized by the kinetic energy associated with the base flow in the rotating frame, $E_{B}$, from (8). The solid black lines are the theoretical growth rates from (15) of the form $e^{2 \sigma t}$ with $K=3$. (d) Same averaged axial kinetic energy $\bar{E}_{z}$ now normalized by $\beta^{2}$. All energies are time-averaged using (17). The black dashed line indicates a constant saturation value. The cases in (c) and (d) are $N 1, \beta=0.01, N 2, \beta=0.03$, $N 3, \beta=0.04, N 4, \beta=0.09$ and $N 5, \beta=0.16$. For all numerical simulations, $E=5 \times 10^{-5}$.

The response of the flow to TDEI and LDEI was also addressed in previous studies examining kinetic energy dissipation. For turbulent flow, the dissipation is expected to scale as $u^{3}$, where $u$ is the velocity (i.e. Kolmogorov 1941). In the numerical studies of the current work, we also find that the dissipation in the bulk scales as $U_{\mathrm{TDEI}}^{3} \sim \mathcal{O}\left(\beta^{3}\right)$. A scaling for boundary layer dissipation is given by $\beta^{2} E^{1 / 2}$ (i.e. Cébron et al. 2010). For the current work and in planetary setting where $\beta \ll E^{1 / 2}$, the bulk dissipation is larger than the boundary layer dissipation. The scaling for the turbulent bulk dissipation is also found in numerical simulations of tidal forcing in a periodic box and in the ellipsoidal geometry with a free surface (e.g. Barker \& Lithwick 2013; Barker et al. 2016). This result is expected since there are no boundaries and thus no viscous dissipation in the boundary. In the study of turbulence generated by LDEI in ellipsoids where both bulk and viscous boundary dissipation are present, the bulk dissipation is also found to be larger than the viscous dissipation. (e.g. Favier et al. 2015).

\subsection{Zonal flows}

Tide-driven forcing can generate steady zonal flows driven by nonlinear self-interactions of the boundary flow that satisfy the no-slip boundary condition. The amplitude of the typically retrograde zonal flows scales with $\beta^{2}$ and is independent of the Ekman number. However, as shown in recent numerical studies of libration by Favier et al. (2015) where the Ekman number is varied, the zonal flow amplitude is increased by the presence of elliptical instability. To look at the influence of the TDEI on the zonal flow, we assume the mean velocity is strongly azimuthal and after subtracting the base flow in (7) and (9), we decompose the flow into its mean azimuthal component in cylindrical coordinates:

$$
\left\langle\overline{U_{\phi}}\right\rangle(r)=\frac{1}{N_{r}} \sum_{z} \sum_{\phi} \sum_{r-\frac{d r}{2}<r^{\prime}<r+\frac{d r}{2}} \overline{U_{\phi}}\left(r^{\prime}, \phi, z\right),
$$

where $N_{r}$ is the number of points averaged in each radial ring. Note that $z$ is fixed for the experiments.

By separating the azimuthal flow into approximately 30 radial rings and averaging the azimuthal velocity in each ring, radial profiles of the time-averaged azimuthal velocities are shown in Fig. 5(a) where the symbols and dotted lines indicate the experimental and DNS measurements respectively. Beginning with $\beta=0.01$ (blue), the retrograde azimuthal flow velocity is quite small. 
(a)

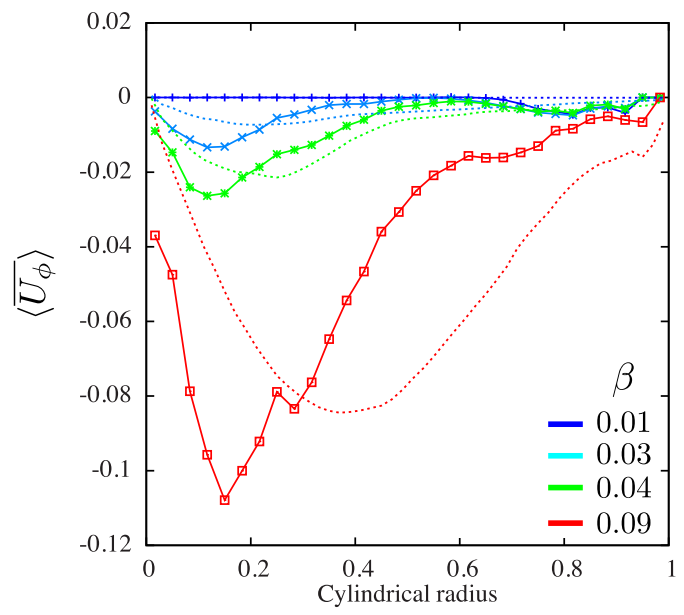

(b)

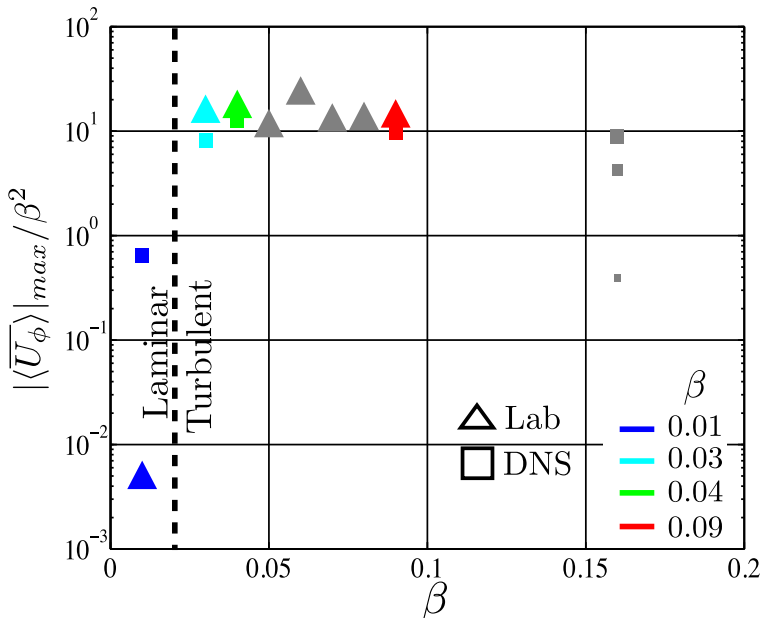

Figure 5. Measurements of tidally driven zonal flows. (a) Radial profiles of the time-averaged dimensionless azimuthal flow velocity, $\left\langle\overline{U_{\phi}}\right\rangle$, with normalized radius $r$. The experimental data at $E=1.5 \times 10^{-5}$ from cases $L 1, \beta=0.01, L 2, \beta=0.03, L 3, \beta=0.04$ and $L 8, \beta=0.09$ are shown with symbols and the DNS cases at $E=5 \times 10^{-5}$ with $N 1, \beta=0.01, N 2, \beta=0.03, N 3, \beta=0.04$ and $N 4, \beta=0.09$ are shown with dotted lines. (b) The maximum velocities, $\left|\left\langle\overline{U_{\phi}}\right\rangle\right|$ max are normalized by $\beta^{2}$ and plotted as a function of $\beta$ where triangles, $(\triangle)$, indicate laboratory experiments and boxes, ( $\square$ ), denote DNS velocity peaks. The coloured symbols for the max values in (b) match the profiles in (a) from which they came. The grey symbols denote additional cases whose profiles were not included in (a). The size of the symbols is inversely proportional to the Ekman number, $E^{-1}$. The vertical dashed line represents the transition from laminar to turbulent flows.

As $\beta$ is increased the amplitude of the retrograde zonal flow grows as well. For all profiles, the experimental data at $E=1.5 \times 10^{-5}$ shows a larger magnitude and more centralized peak than numerical studies at $E=5 \times 10^{-5}$ for the same values of $\beta$. A transition to a stronger more centralized geostrophic flow was also found in studies of LDEI enhanced zonal flow where the Ekman number was decreased and $\beta$ was held constant (i.e. Favier et al. 2015). Thus we believe that the disparity found for zonal flow peaks and locations in the current work may also be related to differences in Ekman number. More generally, the presence of such strong zonal flows generated as the elliptical instability saturates (cf. Favier et al. 2015) may play a crucial role for the long term flow evolution and will be the subject of future studies.

In previous studies of tidal forcing, zonal flow peak amplitudes were shown to scale with $\beta^{2}$ (e.g. Suess 1971; Morize et al. 2010; Sauret et al. 2014). As such, the maximum values of the azimuthal velocity in the radial profiles of Fig. 5(a) are shown as coloured points in Fig. 5(b) normalized by $\beta^{2}$ as a function of $\beta$. The additional grey symbols denote peaks from other profiles excluded in Fig. 5(a) only for clarity. Triangle and square symbols denote experiments and DNS, respectively. The size of the symbols are inversely proportional to the Ekman number, $E^{-1}$, varied between $E=\left[1.5 \times 10^{-5}-5 \times 10^{-4}\right]$.

The dotted vertical line denotes the transition between laminar and TDEI induced turbulent flow around $\beta \sim 0.02$. Below this threshold, no clear trend is revealed and the experimental data at $\beta=0.01$ and zonal flow peaks are on the order of the noise in the system. Above the threshold, the bulk of experimental and numerical data are relatively flat around

$\left|\left\langle\overline{U_{\phi}}\right\rangle\right|_{\max } / \beta^{2} \sim 10$,

indicating that $\beta^{2}$ normalization is well chosen. Additional numerical data at fixed $\beta=0.16$ on the right side of Fig. 5(b) clearly reveal that the amplitude increases as the Ekman number is decreased. However, it is not clear from the present study if this trend continues or the zonal flow saturates at some critical value (cf. Sauret et al. 2014). A summary of the laboratory experimental and numerical zonal flow velocities shown in Fig. 5(b) are compiled in Tables B1 and B2 of Appendix B.

\subsection{Mode coupling and the transition to turbulence}

The transition to turbulence is explored by increasing the strength of the tidal deformation from $\beta \in[0.01-0.16]$ while keeping the tidal frequency fixed at $f_{\text {tide }}=4$ and the Ekman numbers fixed at $E=1.5 \times 10^{-5}, 5 \times 10^{-5}$ for experiments and DNS, respectively.

To characterize the turbulent transition, all velocity components are analysed in the rotating frame at probe points distributed homogeneously in the bulk of the ellipsoid for DNS and in the equatorial plane for the experiments. Because an adaptive time stepping method is used in the DNS, signals must be evaluated on a uniform grid formed using a Langrangian interpolation method. For DNS and experiments, velocity signals are multiplied by a time-periodic Hanning function to ensure all signals are periodic.

A comparison of the power spectrograms from experimental cases $L 1(\beta=0.01), L 3(\beta=0.04)$ and $L 8(\beta=0.09)$ and DNS cases $N 1(\beta=0.01), N 3(\beta=0.04)$ and $N 4(\beta=0.09)$ are shown in Fig. 6. Sliding window discrete Fourier transforms are performed on a moving window of 10 spin periods with a 90 per cent overlap. The first signal is the strong peak at $f=4$ corresponding to the $f_{\text {tide }}=4$ of the tidal forcing that drives the ellipsoidal base flow and persists for all times. The second shared signal is the zonal flow at $f=0$ whose characteristics are discussed in Section 5.2.

From side-view visualizations and from the flow energetics provided in Fig. 4, the case $\beta=0.01$ shown in Figs 6(a) and (b) is stable, exhibiting no signs of bulk turbulence for all times and one strong peak at the tidal forcing, $f_{\text {tide }}=4$. Figs $6(\mathrm{c})$ and (d) show the spectrogram for $\beta=0.04$. Following the onset of the tidal forcing, after a certain amount of time that depends on the strength of the tidal forcing and Ekman number, a strong persistent signal appears at $f=2$, associated with the excitation of primary inertial modes at half of the forcing frequency. 

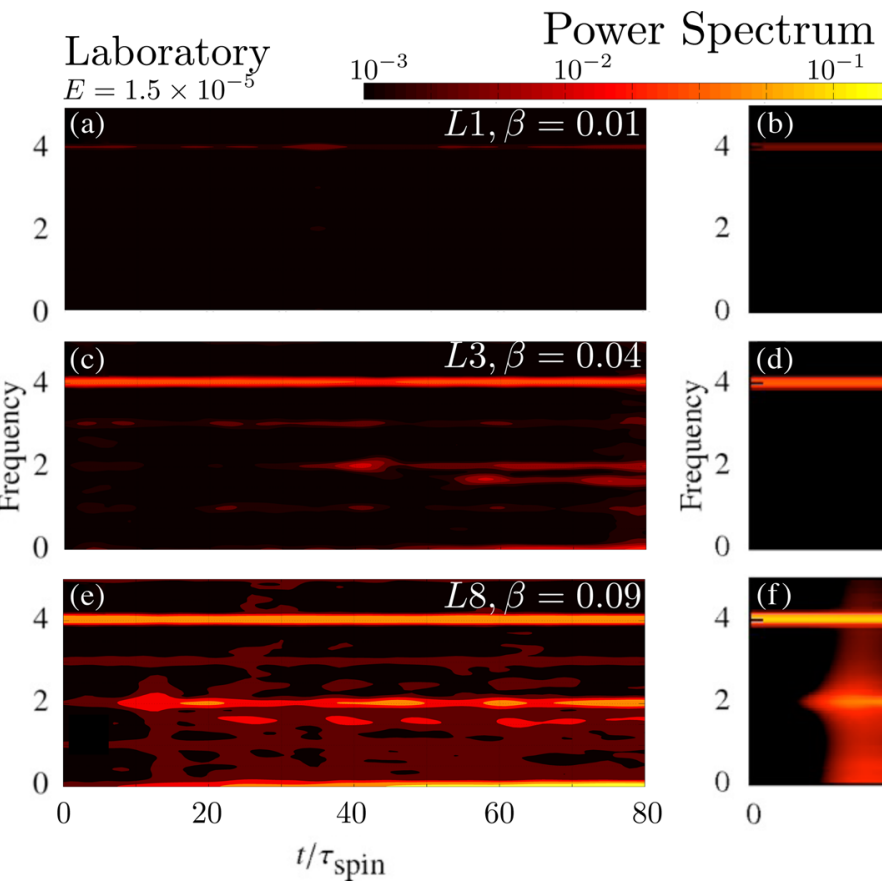
$10^{-1}$ $10^{0}$

DNS

\section{(b)} $E=5 \times 10^{-5}$

2

0
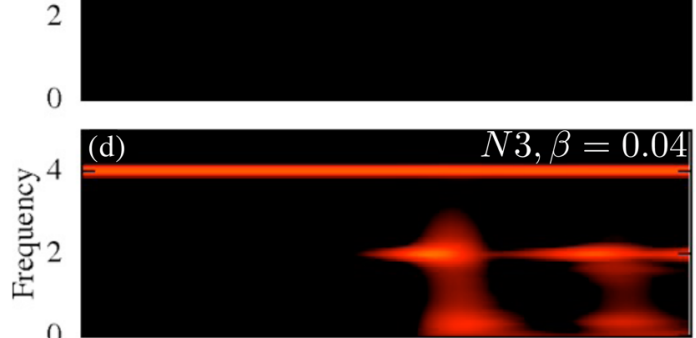

(d)
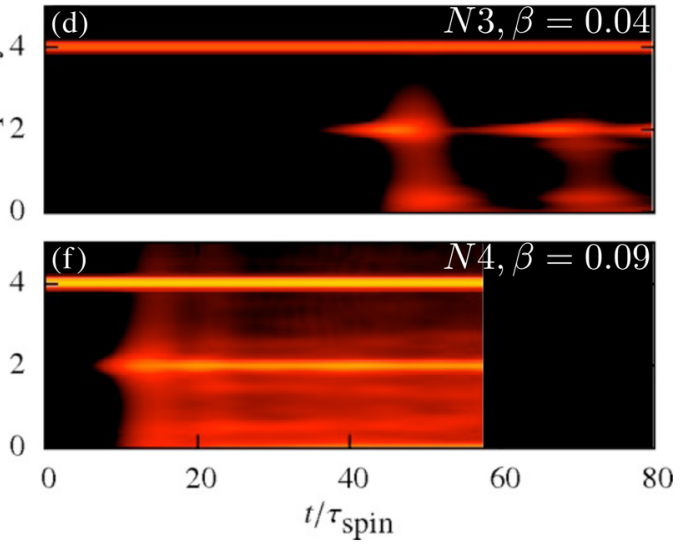

Figure 6. Tidal forcing power spectrograms showing the dominant flow frequencies. Shown are cases (a) $L 1, \beta=0.01$, (b) $N 1, \beta=0.01$, (c) $L 3, \beta=0.04$, (d) $N 3, \beta=0.04$, (e) $L 8, \beta=0.09$ and (f) $N 4, \beta=0.09$ where $f_{\text {tide }}=4$ and the Ekman number is fixed at $E=1.5 \times 10^{-5}, 5 \times 10^{-5}$ for the laboratory experiments and DNS, respectively.

The existence of these inertial modes at $f=2$ satisfies the temporal resonance condition in (11). Spatially, the modes are identifiable by their axial pancake-like structures as shown in the middle images of Figs 3(a) and (b). Although we have not explicitly determined the wavenumbers of these modes, nearly identical modes have also been found in laboratory experiments and numerical simulations of LDEI (i.e. Grannan et al. 2014; Favier et al. 2015). In those studies, the inertial modes have an eigenfrequency $\left|f_{\text {tide }} / 2\right| \lesssim 2$ and their spatial structure is characterized by both an azimuthal wavenumber, $m_{1,2}=1$, and a large number of stacked axial structures. Analytical descriptions of these ellipsoidal modes are theoretically possible using a polynomial description of the modes but difficult in practice because of the need to use high degree polynomials to resolve these high axial wavenumber modes (i.e. Vantieghem 2014). Instead analytical solutions from the sphere and polar flattened spheroid have been implemented and show the existence of $m_{1,2}=1$ that satisfies the spatial resonance condition in (12) and have high frequencies at $\lambda \sim 1.9$ (i.e. Grannan et al. 2014; Favier et al. 2015). Thus, we contend that similar inertial modes with the same spatial description exist in the ellipsoidal geometry of the current work at an adjusted frequency, $\left|\lambda_{1,2}\right| \sim 2$.

As the tidal deformation is increased again, the growth rate given by (15) also increases and thus the TDEI for $\beta=0.09$, shown in Figs 6(e) and (f), occurs earlier in time. For both cases $\beta=0.04$, 0.09 , after the amplitude of the primary inertial modes has grown, secondary resonances can occur whose driving force is the primary inertial mode frequency $f=2$, instead of the tidal forcing at $f=4$.

Evidence for these secondary resonances is seen more clearly in the full time-averaged frequency spectrum shown for experiments and numerics in Figs 7(a) and (b), respectively. We first note strong spectral peaks at frequencies $f=1,2,3$ are found only in the experiments, and non-existent in the DNS. For $\beta=0.01$ in Fig. 7(a), the signals at $f=1,2,3$ may have two causes. The first may be periodic light intensity changes occurring as the camera, in the rotating frame, moves between different laser light sources and the mirrors used for reflecting the light mounted in the lab frame.

The second cause may be a misalignment of the rotating container or the rollers replicating the tidal deformation. In any case, these signals are related to the harmonics associated with the rotating frequencies $\left|\bar{\Omega}_{\text {spin }}, \Omega_{\text {orb }}\right| \sim 1 \mathrm{~Hz}$ and are found in all the experimental measurements. However, the good agreement with the DNS suggests that these signals do not appear to noticeably affect the results and the peaks are still quite small in comparison to the primary forcing at $f_{\text {tide }}=4$ for cases above the stable one at $\beta=0.01$.

Concerning the secondary modes excited by the primary inertial modes at $f=2$, although the spatial structures for these secondary modes have not been uniquely determined, peak frequencies at $f \sim 1.5$ and $f \sim 0.5$ are still evident in Fig. 7(b). The frequency condition (11) is satisfied for a secondary resonance being driven by the primary inertial mode at $f_{\text {tide }} / 2=2$. For the horizontal spectra from the experiments in Fig. 7(a), the only clear peak resides at $f \sim 1.5$ while Fig. 7(b) shows a second peak at $f \sim 0.5$. Similar to the method used in libration simulations from Favier et al. (2015), a decomposition of the tidal simulation spectra into axial and horizontal components (not shown) reveals that the mode at $f \sim 0.5$ is characterized by strong axial motions untraceable in the experimental data. The mode at $f \sim 1.5$ is composed of strong horizontal motions and visible by both methods.

Finally, a comparison of the full power spectra generated through TDEI and LDEI is shown in Figs 8(a) and (b) respectively. Fig. 8(a) displays cases $L 8\left(\beta=0.09, E=1.5 \times 10^{-5}\right.$, blue $)$ and $N 4(\beta=0.09$, $E=5 \times 10^{-5}$, red) from the current work. Fig. 8(b) is reproduced from the experimental (blue) and DNS (red) spectra in the LDEI studies of Favier et al. (2015) where $f_{\text {lib }}=4, \epsilon_{\mathrm{lib}}=0.8$ and $\beta=0.34$ are fixed and $E=2.7 \times 10^{-5}$ and $E=10^{-4}$ for the experiments and numerics, respectively. In both images the forcing, primary inertial modes, secondary inertial modes and high frequency tails are nearly identical. Note that the experimental data has been cut at $f \sim 8$ where 
(a) Laboratory

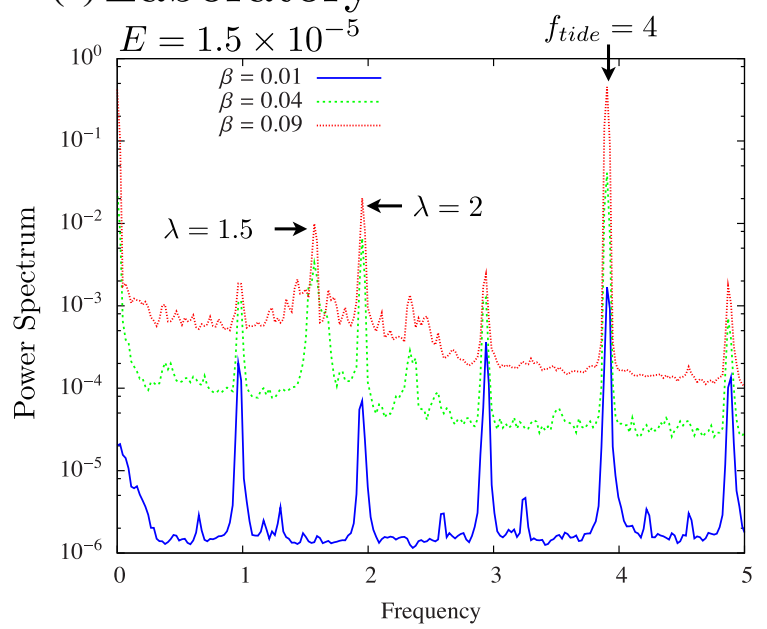

(b) DNS

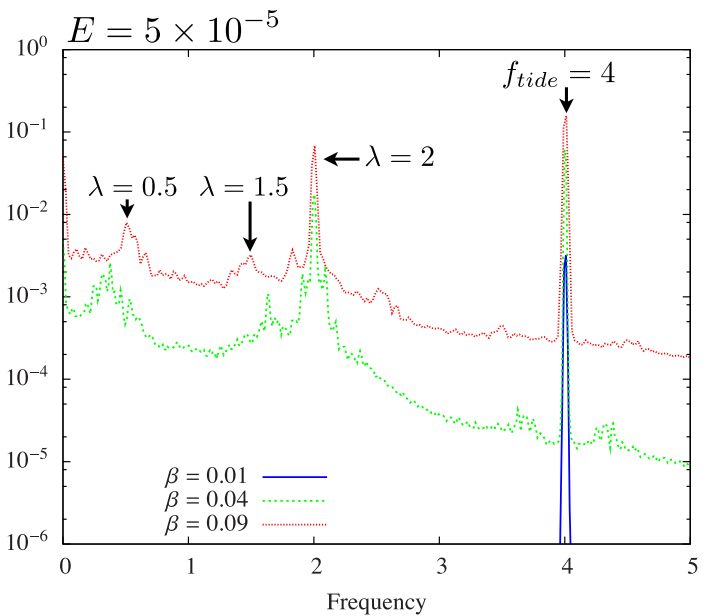

Figure 7. The time-averaged tidal forcing power spectrum of the saturated turbulent flow. (a) Laboratory experiments $L 1, \beta=0.01, L 3, \beta=0.04$ and $L 8$, $\beta=0.09$ where Ekman, $E=1.5 \times 10^{-5}$. (b) A similar power spectrum from DNS cases $N 1, \beta=0.01, N 3, \beta=0.04$ and $N 4, \beta=0.09$ where $E=5 \times 10^{-5}$.

(a) Tides

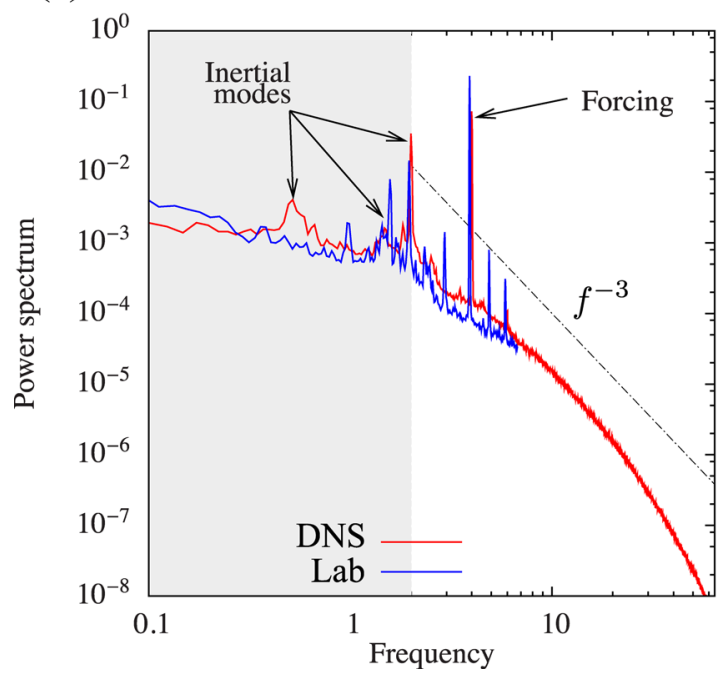

(b) Libration

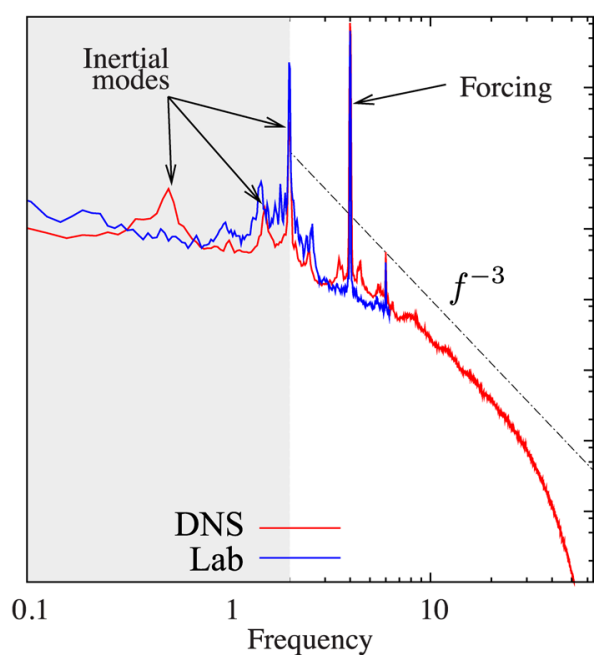

Figure 8. (a) The time-averaged power spectrum of the saturated turbulent flow in the current tidal studies where $f_{\text {tide }}=4$ and $\beta=0.09$. The laboratory case, $L 8$, where $E=1.5 \times 10^{-5}$ is shown in blue. The numerical case, $N 4$, where $E=5 \times 10^{-5}$ is shown in red. (b) The time-averaged power spectrum of the saturated turbulent flow in the libration studies where $\epsilon_{\text {lib }}=0.8$ and $f_{\text {lib }}=4$. The laboratory case $V$ from Grannan et al. (2014) where $E=2.7 \times 10^{-5}$ is shown in blue. The DNS case $A 6$ from Favier et al. (2015) where $E=10^{-4}$ is shown in red. The dashed line has an $f^{-3}$ slope.

the spectra is $\mathcal{O}\left(10^{-5}\right)$. At greater frequencies the signal to noise ratio is unity in the experimental data and only the numerical spectra fills the high frequency tail.

For both tides and libration the majority of the energy is contained in the forcing and excited inertial modes. The power in the higher frequency spectra more closely follows an $f^{-3}$ scaling indicative of scalings for rotating turbulence wherein the presence of the Coriolis force reduces the forward energy cascade. The same scalings were also found for the wavenumbers in the spatial spectra of local models of tidal forcing and simulations of libration in ellipsoids (i.e. Barker \& Lithwick 2013; Favier et al. 2015; Barker et al. 2016).

\section{CONCLUSIONS}

We have combined laboratory experiments with numerical simulations to show, for the first time, that tides can mechanically drive bulk filling turbulence in an interior fluid region via elliptical in- stability (see Figs $3 a$ and $b$ ). We find strong agreement with the theoretical prediction for the growth rate and the subsequent instability whose forcing and inertial mode frequencies satisfy the resonance conditions for the TDEI (see Fig. 4). The transition to turbulence is characterized first by the growth of primary inertial modes, then the excitation of additional secondary inertial modes, and finally saturated bulk turbulent flow (see Figs 6 and 7). Furthermore, our tidal results have both qualitative and quantitative similarities to librationally driven flows (see Figs 3c, d and 8).

Tidal deformation of an elastically deformed ellipsoid and longitudinal libration of an ellipsoid with a rigid boundary represent two end-member cases for the geophysically relevant periodic mechanical forcings that many planetary bodies experience. Our tidal case better describes a flexible icy shell, for instance, in a nonsynchronous orbit, where the orbital and spin rate of the body are unequal and the elastic boundary responds quickly to tidal distortions. Our libration case is more relevant for planetary bodies whose shapes have some degree of intrinsic ellipsoidal distortion 
[e.g. Vesta, Ermakov et al. (2014), and/or are in so-called synchronous orbits where the time-averaged tidal forcing generates the ellipsoidal deformation.

Although tidal and librational forcing mechanisms are quite different, their mathematical formulation can be generalized in the same way as shown in Section 2 and Tables 1 and 2. The strong agreement found between the transition to, and characteristics of, the bulk turbulence driven by tides and libration hints at a generic response of the fluid interior to elliptical instability as illustrated in Figs 3 and 8, showing side-view visualizations and power spectra, respectively. Furthermore, the small-scale turbulence observed is more easily excited as the Ekman number is decreased (e.g. Lacaze et al. 2004; Le Bars et al. 2010) and may be similar to the smallscale turbulence that might be generated in planetary fluid interiors where $E \ll 1$.

We find that the saturated turbulent velocity induced by TDEI scales linearly with $U_{\text {TDEI }} \sim \beta$. In comparison, a bulk azimuthal zonal flow is shown to scale with $\beta^{2}$. In planetary settings where $\beta<10^{-4}$ (e.g. Cébron et al. 2012a), the turbulent velocity scaling is therefore much larger than that of the zonal flow and may be of critical importance for planetary processes like tidal dissipation in subsurface oceans and magnetic field generation in liquid metal cores.

To investigate the generation of turbulence in planetary bodies based on the velocity scaling in (20), we define a Reynolds number that describes the strength of turbulence in a flow, $R e=U R / v$, where $U$ is the dimensional turbulent velocity, $R$ is the outer radius of the fluid layer and $v$ is the kinematic viscosity. Using (20) a dimensional velocity, $U \sim U_{\mathrm{TDEI}} \bar{\Omega}_{\text {spin }} R$, is formed. The Reynolds number can then be rewritten in terms of the mechanical forcing parameters such that

$R e=\frac{\beta}{E}$.

For elliptical instability to occur, it is required that $\sigma>0$ in (15) and thus

$\frac{\sigma_{\text {inv }}}{\sqrt{E}} \sim \frac{\epsilon_{\text {tide, lib }} \beta}{\sqrt{E}} \geq 1$

This condition for elliptical instability in (24) can be rewritten in terms of the Reynolds number in (23) forming a critical Reynolds number, $R e_{\mathrm{cr}}$ :

$R e_{\text {cr }}=\frac{1}{\epsilon_{\text {tide, lib }} \sqrt{E}}$.

Thus the condition for the onset of elliptical instability in (24) can be redefined as $R e / R e_{\mathrm{cr}}>1$. For the subsurface ocean on Europa, the parameters $\epsilon=2 \times 10^{-4}, \beta=9.7 \times 10^{-4}$ and $E=2 \times 10^{-14}$ are taken from Cébron et al. (2012a). Using the planetary values for Europa, $R e_{\mathrm{cr}} \sim 4 \times 10^{10}$ while $R e \sim 5 \times 10^{10}$ indicating that the body is marginally unstable and mechanical forcing may be capable of generating turbulent flow that could play an important geophysical role.

New observations of Enceladus have shown the presence of a large libration in addition to a global subsurface ocean [i.e. Cadek et al. (2016); Thomas et al. (2016)]. For Enceladus, $\epsilon_{\text {lib }}=2 \times 10^{-3}$, $\beta=9 \times 10^{-3}$ following the formulation used in Cébron et al. (2012a), and $E=3 \times 10^{-13}$ revealing that $R e_{c r}=9 \times 10^{8}$ and $R e=3 \times 10^{10}$ suggesting that the mechanical response of the fluid may drive significant flows in a subsurface ocean.

Currently, most models for tidal dissipation only consider viscoelastic dissipation in a given body's solid layers, such as icy shells. These models ignore the effects of dissipation within lower viscosity fluid regions (e.g. Williams \& Boggs 2015). Recent models have now begun to include dissipation due to direct tidal resonances in subsurface oceans on icy bodies (e.g. Tyler 2008, 2014; Chen et al. 2014; Matsuyama 2014; Kamata et al. 2015). In the future we will extend our models to estimate how tidal and librational elliptical instabilities can drive further dissipation in low viscosity planetary fluid layers [cf., Ibragimov (2007) for Earth's oceans].

In addition, advance laboratory experiments currently under construction and high resolution numerical simulations using full ellipsoids and local Cartesian geometries will focus on elliptical instability with the inclusion of inner cores, fluid stratification and multiple forcing frequencies at even more extreme ranges of forcing parameters. These studies will aid in investigating whether the scaling for the velocity, like that proposed in (20), holds, changes, or saturates at some critical value of the parameters used. Recent precession and tidal studies at more extreme parameters (i.e. Barker \& Lithwick 2013, 2014; Lin et al. 2015) have shown the formation of large scale structures similar to those being investigated in convection (i.e. Julien et al. 2012; Stellmach et al. 2014; Favier et al. 2014b; Guervilly et al. 2014; Aurnou et al. 2015; Guervilly et al. 2015; Plumley et al. 2016). Furthermore, precession, nutation, tides, libration and even small body impacts, do not work in isolation but are experienced in varying degrees by all bodies. For instance, the combined effect of tidal and precessional forcing, as seen in Morize et al. (2010), permits rich dynamics. Thus, there may be many ways for mechanical forcing to perturb the fluid motions in planetary interiors.

\section{ACKNOWLEDGEMENTS}

AMG gratefully acknowledges the financial support of the Chateaubriand Fellowship Program for his 9 month stay in Marseille, France. AMG and JMA gratefully acknowledge financial support from the National Science Foundation (NSF) Geophysics Program for salary support and from the NASA PG\&G (Planetary Geology and Geophysics) Program for funding the fabrication of the UCLA libration device. MLB gratefully acknowledges financial support from the ANR-JCJC-SIMI5 program (Proposal ANR-13-JS05-0004-01). BF gratefully acknowledges support from IDRIS (Institut du Developpement et des Ressources en Informatique Scientifique) for computational time on Turing (Project Nos 100508 and 100614) and from the HPC resources of Aix-Marseille Université financed by the project Equip@Meso (No. ANR-10EQPX-29-01) of the program Investissements d'Avenir supervised by the Agence Nationale pour la Recherche (Project Nos 15 b011 and $16 \mathrm{~b} 020)$.

\section{REFERENCES}

Anderson, J.D., Sjogren, W.L. \& Schubert, G., 1996. Galileo gravity results and the internal structure of Io, Science, 272, 709-712.

Anderson, J.D., Schubert, G., Jacobson, R.A., Lau, E.L., Moore, W.B. \& Sjogren, W.L., 1998. Europa's differentiated internal structure: inferences from four Galileo encounters, Science, 281(5385), 2019-2022.

Aurnou, J.M., Calkins, M.A., Cheng, J.S., Julien, K., King, E.M., Nieves, D., Soderlund, K.M. \& Stellmach, S., 2015. Rotating convective turbulence in Earth and planetary cores, Phys. Earth planet. Inter., 246, 52-71.

Barker, A.J. \& Lithwick, Y., 2013. Non-linear evolution of the tidal elliptical instability in gaseous planets and stars, Mon. Not. R. Astron. Soc., 435(4), 3614-3626.

Barker, A.J. \& Lithwick, Y., 2014. Non-linear evolution of the elliptical instability in the presence of weak magnetic fields, Mon. Not. R. Astron. Soc., 437, 305-315. 
Barker, A.J., Braviner, H.J. \& Ogilvie, G.I., 2016. Non-linear tides in a homogeneous rotating planet or star: global modes and elliptical instability, Mon. Not. R. Astron. Soc., 459(1), 924-938.

Busse, F., 2010. Mean zonal flows generated by librations of a rotating spherical cavity, J. Fluid Mech., 650, 505-512.

Cadek, O. et al., 2016. Enceladus' internal ocean and ice shell constrained from Cassini gravity, shape, and libration data, Geophys. Res. Lett., 43(11), 5653-5660.

Calkins, M., Noir, J., Eldredge, J. \& Aurnou, J., 2010. Axisymmetric simulations of libration-driven fluid dynamics in a spherical shell geometry, Phys. Fluids, 22, 1-12.

Cébron, D. \& Hollerbach, R., 2014. Tidally driven dynamos in a rotating sphere, Astrophys. J. Lett., 789(1), L25:1-5.

Cébron, D., Maubert, P. \& Le Bars, M., 2010. Tidal instability in a rotating and differentially heated ellipsoidal shell, Geophys. J. Int., 182(3), 13111318.

Cébron, D., Le Bars, M., Moutou, C. \& Le Gal, P., 2012a. Elliptical instability in terrestrial planets and moons, Astron. Astrophys., 539, A78:1-16.

Cébron, D., Le Bars, M., Noir, J. \& Aurnou, J.M., 2012b. Libration driven elliptical instability, Phys. Fluids, 24, 061703:1-7.

Chan, K., Liao, X. \& Zhang, K., 2011. Simulations of fluid motions in ellipsoidal planetary cores driven by longitudinal libration, Phys. Earth planet. Inter., 187, 139-403.

Chen, E. M.A., Nimmo, F. \& Glatzmaier, G.A., 2014. Tidal heating in icy satellite oceans, Icarus, 229, 11-30.

Comstock, R.L. \& Bills, B.G., 2003. A solar system survey of forced librations in longitude, J. geophys. Res., 108, 1-13.

Egbert, G.D. \& Ray, R.D., 2003. Semi-diurnal and diurnal tidal dissipation from TOPEX/Poseidon altimetry, Geophys. Res. Lett., 30(17), doi:10.1029/2003GL017676.

Ermakov, A.I., Zuber, M.T., Smith, D.E., Raymond, C.A., Balmino, G., Fu, R.R. \& Ivanov, B.A., 2014. Constraints on Vesta's interior structure using gravity and shape models from the Dawn mission, Icarus, 240, 146-160.

Favier, B., Barker, A.J., Baruteau, C. \& Ogilvie, G.I., 2014a. Non-linear evolution of tidally forced inertial waves in rotating fluid bodies, Mon. Not. R. Astron. Soc., 439, 845-860.

Favier, B., Silvers, L.J. \& Proctor, M.R.E., 2014b. Inverse cascade and symmetry breaking in rapidly rotating Boussinesq convection, Phys. Fluids, 9, 096605:1-22.

Favier, B., Grannan, A.M., Le Bars, M. \& Aurnou, J.M., 2015. Generation and maintenance of bulk turbulence by libration-driven elliptical instability, Phys. Fluids, 27(6), 066601.

Fischer, P.F., Loth, F., Lee, S.E., Lee, S.-W., Smith, D.S. \& Bassiouny, H.S., 2007. Simulation of high-Reynolds number vascular flows, Comput. Methods Appl. Mech. Eng., 196(31), 3049-3060.

Fu, R.R. et al., 2012. An ancient core dynamo in asteroid Vesta, Science, 338, 238-241.

Garrick-Bethell, I., Weiss, B.P., Shuster, D.L. \& Buz, J., 2009. Early lunar magnetism, Science, 323(5912), 356-359.

Grannan, A.M., Le Bars, M., Cébron, D. \& Aurnou, J.M., 2014. Experimental study of global-scale turbulence in a librating ellipsoid, Phys. Fluids, 26, 126601:1-19.

Greenspan, H.P., 1969. The Theory of Rotating Fluids, Cambridge Univ. Press.

Greff-Lefftz, M., Métivier, L. \& Legros, H., 2005. Analytical solutions of Love numbers for a hydrostatic ellipsoidal incompressible homogeneous Earth, Celest. Mech. Dyn. Astron., 93(1-4), 113-146.

Guervilly, C., Hughes, D.W. \& Jones, C.A., 2014. Large-scale vortices in rapidly rotating Rayleigh-Bénard convection, J. Fluid Mech., 758, 407435.

Guervilly, C., Hughes, D.W. \& Jones, C.A., 2015. Generation of magnetic fields by large-scale vortices in rotating convection, Phys. Rev. E, 91, 041001:1-5.

Ibragimov, R.N., 2007. Oscillatory nature and dissipation of of the internal waves energy spectrum in the deep ocean, Eur. Phys. J. Appl. Phys., 40(3), $315-334$.
Johnson, C.L. et al., 2015. Low-altitude magnetic field measurements by MESSENGER reveal Mercury's ancient crustal field, Science, 348(6237), 892-895.

Jones, C.A., 2011. Planetary magnetic fields and fluid dynamos, Annu. Rev. Fluid Mech., 43(1), 583-614.

Julien, K., Rubio, A.M., Grooms, I. \& Knobloch, E., 2012. Statistical and physical balances in low-Rossby-number Rayleigh-Bénard convection, Geophys. Astrophys. Fluid Dyn., 106(4-5), 392-428.

Kamata, S., Matsuyama, I. \& Nimmo, F., 2015. Tidal resonance in icy satellites with subsurface oceans, J. geophys. Res., 120(9), 1528-1542.

Kerswell, R.R., 2002. Elliptical instability, Annu. Rev. Fluid Mech., 34, $83-113$.

Kerswell, R.R. \& Malkus, W.V.R., 1998. Tidal instability as the source for Io's magnetic signature, Geophys. Res. Lett., 25(5), 603-606.

Kolmogorov, A.N., 1941. The local structure of turbulence in incompressible viscous fluid for very large Reynolds numbers, in Dokl. Akad. Nauk SSSR, 30, 301-305.

Lacaze, L., Le Gal, P. \& Le Dizes, S., 2004. Elliptical instability in a rotating spheroid, J. Fluid Mech., 505, 1-22.

Lacaze, L., Le Gal, P. \& Le Dizes, S., 2005. Elliptical instability of the flow in a rotating shell, Phys. Earth planet. Inter., 151, 194-205.

Le Bars, M., Le Dizes, S. \& Le Gal, P., 2007. Coriolis effects on the elliptical instability in cylindrical and spherical rotating containers, J. Fluid Mech., $\mathbf{5 8 5}, 323-342$.

Le Bars, M., Lacaze, L., Le Dizes, S., Le Gal, P. \& Rieutord, M., 2010. Tidal instability in stellar and planetary binary systems, Phys. Earth planet. Inter., 178, 48-55.

Le Bars, M., Cébron, D. \& Le Gal, P., 2015. Flows driven by libration, precession, and tides, Annu. Rev. Fluid Mech., 47, 163-194.

Le Dizes, S., 2000. Three-dimensional instability of a multipolar vortex in a rotating flow, Phys. Fluids, 12, 2762-2774.

Lin, Y., Marti, P. \& Noir, J., 2015. Shear-driven parametric instability in a precessing sphere, Phys. Fluids, 27(4), 046601:1-17.

Matsuyama, I., 2014. Tidal dissipation in the oceans of icy satellites, Icarus, 242, 11-18.

Meunier, P. \& Lewecke, T., 2003. Analysis and treatment of errors due to high velocity gradients in particle image velocimetry, Exp. Fluids, 35, 408-421.

Morize, C., Le Bars, M., Le Gal, P. \& Tilgner, A., 2010. Experimental determination of zonal winds driven by tides, Phys. Rev. Lett., 104, 214501:1-4.

Nimmo, F., 2009. Energetics of asteroid dynamos and the role of compositional convection, Geophys. Res. Lett., 36, L10201:1-6.

Noir, J., Calkins, M., Lasbleis, M., Cantwell, J. \& Aurnou, J.M., 2010. Experimental study of libration-driven zonal flows in a straight cylinder, Phys. Earth planet. Inter., 182, 98-1106.

Noir, J., Cébron, D., Le Bars, M., Sauret, A. \& Aurnou, J.M., 2012. Experimental study of libration-driven zonal flows in non-axisymmetric containers, Phys. Earth planet. Inter., 204-205, 1-10.

Olson, P., 2013. The new core paradox, Science, 342(6157), 431-432.

O'Rourke, J.G. \& Stevenson, D.J., 2016. Powering Earth's dynamo with magnesium precipitation from the core, Nature, 529(7586), 387-389.

Plumley, M., Julien, K., Marti, P. \& Stellmach, S., 2016. The effects of Ekman pumping on quasi-geostrophic Rayleigh-Bénard convection, $J$. Fluid Mech., 803, 51-71.

Pozzo, M., Davies, C., Gubbins, D. \& Alfé, D., 2012. Thermal and electrical conductivity of iron at Earth's core conditions, Nature, 485, 355-358.

Sauret, A., 2012. Forçage Harmonique D'Écoulements en Rotation: Vents Zonaux, Ondes Inertielles et Instabilités, PhD thesis, Universite AixMarseille.

Sauret, A., Cébron, D., Morize, C. \& Le Bars, M., 2010. Experimental and numerical study of mean zonal flows generated by librations of a rotating spherical cavity, J. Fluid Mech., 662, 260-268.

Sauret, A., Le Bars, M. \& Le Gal, P., 2014. Tide-driven shear instability in planetary liquid cores, Geophys. Res. Lett., 41, 6078-6083.

Schubert, G. \& Soderlund, K., 2011. Planetary magnetic fields: observations and models, Phys. Earth planet. Inter., 187, 92-108.

Schubert, G., Anderson, J., Spohn, T. \& Mckinnon, W., 2004. Interior composition, structure and dynamics of the Galilean satellites, in Jupiter: The 
Planet, Satellites and Magnetosphere, vol. 1, pp. 281-306, eds Bagenal, F., Dowling, T.E. \& McKinnon, W.B., Cambridge Univ. Press.

Stark, A. et al., 2015. First MESSENGER orbital observations of Mercury's librations, Geophys. Res. Lett., 42, 1-9.

Stellmach, S., Lischper, M., Julien, K., Vasil, G., Cheng, J.S., Ribeiro, A., King, E.M. \& Aurnou, J.M., 2014. Approaching the asymptotic regime of rapidly rotating convection: boundary layers versus interior dynamics, Phys. Rev. Lett., 113, 254501:1-5.

Suess, S., 1971. Viscous flow in a deformable rotating container, J. Fluid Mech., 45, 189-201.

Tarduno, J.A. et al., 2012. Evidence for a dynamo in the main group pallasite parent body, Science, 338, 93-95.

Thomas, P.C., Tajeddine, R., Tiscareno, M.S., Burns, J.A., Joseph, J., Loredo, T.J., Helfenstein, P. \& Porco, C., 2016. Enceladus' measured physical libration requires a global subsurface ocean, Icarus, 264, 37-47.

Tilgner, A., 2005. Precession driven dynamos, Phys. Fluids, 17, 034104:1-6.

Tyler, R.H., 2008. Strong ocean tidal flow and heating on moons of the outer planets, Nature, 456, 770-772.

Tyler, R.H., 2014. Comparative estimates of the heat generated by ocean tides on icy satellites in the outer Solar System, Icarus, 243(0), 358-385.

Van Hoolst, T., Baland, R.-M. \& Trinh, A., 2013. On the librations and tides of large icy satellites, Icarus, 226, 299-315.

Vantieghem, S., 2014. Inertial modes in a rotating triaxial ellipsoid, Proc. R. Soc. A, 470(2168), 20140093, 1-22.

Wang, C.-Y., 1970. Cylindrical tank of fluid oscillating about a state of steady rotation, J. Fluid Mech., 41, 581-592.

Wei, X., Arlt, R. \& Tilgner, A., 2014. A simplified model of collision-driven dynamo action in small bodies, Phys. Earth planet. Inter., 231, 30-38.

Williams, J.G. \& Boggs, D., 2015. Tides on the moon: Theory and determination of dissipation, J. geophys. Res., 120(4), 689-724.

Wu, C. \& Roberts, P.H., 2009. On a dynamo driven by topographic precession, Geophys. Astrophys. Fluid Dyn., 13, 467-501.

Wu, C. \& Roberts, P.H., 2013. On a dynamo driven topographically by longitudinal libration, Geophys. Astrophys. Fluid Dyn., 107, $20-44$.

Yoder, C.F., Konopliv, A.S., Yuan, D.N., Standish, E.M. \& Folkner, W.M., 2003. Fluid core size of Mars from detection of the solar tide, Science, 300(5617), 299-303.

Zhang, K., Chan, K. \& Liao, X., 2011. On fluid motion in librating ellipsoids with moderate equatorial eccentricity, J. Fluid Mech., 673, 468-479.

Zhang, P., Cohn, R.E. \& Haule, K., 2015. Effects of electron correlations on transport properties of iron at earth's core conditions, Nature, 517, $605-607$.

\section{APPENDIX A: BOUNDARY CONDITION IN TIDE SIMULATIONS}

In the orbital frame, the elliptical distortion is fixed and the boundary maintains a constant horizontal velocity tangent to the ellipsoidal surface. At any given height $-c<z<c$, the horizontal shape of the container is given by

$\frac{x^{2}}{a^{\prime 2}}+\frac{y^{2}}{b^{\prime 2}}=1$,

with $a^{\prime}=a \sqrt{1-z^{2} / c^{2}}$ and $b^{\prime}=b \sqrt{1-z^{2} / c^{2}}$. This curve can be parametrized by

$\mathbf{X}(s)=\left(\begin{array}{c}x=a^{\prime} \cos s \\ y=b^{\prime} \sin s \\ z\end{array}\right), \quad$ with $\quad s \in[0-2 \pi]$ with a normalized tangent vector given by

$$
\begin{aligned}
\boldsymbol{\tau}=\frac{\mathbf{X}_{s}}{\left|\mathbf{X}_{s}\right|} & =\frac{1}{\sqrt{a^{\prime 2} \sin ^{2} s+b^{\prime 2} \cos ^{2} s}}\left(\begin{array}{c}
-a^{\prime} \cos s \\
b^{\prime} \sin s \\
0
\end{array}\right) \\
& =\frac{1}{\sqrt{a^{2} y^{2} / b^{2}+b^{2} x^{2} / a^{2}}}\left(\begin{array}{c}
-a y / b \\
b x / a \\
0
\end{array}\right) .
\end{aligned}
$$

The boundary condition for the velocity on the elliptical boundary is finally

$\mathbf{u}_{\text {b.c. }}=(1-\gamma) \sqrt{\frac{a^{\prime 2}+b^{\prime 2}}{2}} \boldsymbol{\tau}$.

\section{APPENDIX B: PARAMETERS FROM LABORATORY EXPERIMENTAL AND NUMERICAL STUDIES}

Table B1. Tidal laboratory experimental cases signified by $(L-)$. For all cases, the Ekman number, $E=$ $\nu /\left(\bar{\Omega}_{\text {spin }} R^{2}\right)=1.5 \times 10^{-5}$ and the ratio of orbital rate the spin rate, $\gamma_{\text {tide }}=-1$, are fixed. The equatorial ellipticity is $\beta=\left(a^{2}-b^{2}\right) /\left(a^{2}+b^{2}\right)$. Case $L 1$ is TDEI stable (No) and all other cases are TDEI unstable (Yes). $\left|\left\langle\bar{U}_{\phi}\right\rangle\right|_{\max }$ is the maximum absolute value for the time and spatially averaged zonal flow defined in eq. (21) and plotted in Fig. 5.

\begin{tabular}{lccc}
\hline Case & $\beta$ & TDEI & $\left|\overline{U_{\phi}}\right|_{\max }$ \\
\hline$L 1$ & 0.01 & No & 0.0046 \\
$L 2$ & 0.03 & Yes & 0.0133 \\
$L 3$ & 0.04 & Yes & 0.0263 \\
$L 4$ & 0.05 & Yes & 0.0269 \\
$L 5$ & 0.06 & Yes & 0.0795 \\
$L 6$ & 0.07 & Yes & 0.0597 \\
$L 7$ & 0.08 & Yes & 0.0808 \\
$L 8$ & 0.09 & Yes & 0.1079 \\
\hline
\end{tabular}

Table B2. Tidal DNS cases signified by $(N-)$. For all cases, the number of elements, $\mathcal{E}=3200$, and the ratio of orbital rate to the spin rate, $\gamma_{\text {tide }}=-1$, are fixed. $N$ is the order of the Legendre polynomials used in the simulations, $\beta=\left(a^{2}-b^{2}\right) /\left(a^{2}+b^{2}\right)$, and $E=v /\left(\bar{\Omega}_{\text {spin }} R^{2}\right)$ is the Ekman number. Case $N 1$ is TDEI stable (No) and all other cases are TDEI unstable (Yes). $\left|\left\langle\bar{U}_{\phi}\right\rangle\right|_{\max }$ is the absolute maximum value for the time and spatially averaged zonal flow defined in eq. (21) and plotted in Fig. 5.

\begin{tabular}{lccccc}
\hline Case & $E$ & $N$ & $\beta$ & TDEI & $\left|\left\langle\overline{U_{\phi}}\right\rangle\right|_{\max }$ \\
\hline$N 1$ & $5.0 \times 10^{-5}$ & 17 & 0.01 & No & $6.5 \mathrm{e}-5$ \\
$N 2$ & $5.0 \times 10^{-5}$ & 17 & 0.03 & Yes & 0.0073 \\
$N 3$ & $5.0 \times 10^{-5}$ & 17 & 0.04 & Yes & 0.0202 \\
$N 4$ & $5.0 \times 10^{-5}$ & 17 & 0.09 & Yes & 0.0791 \\
$N 5$ & $5.0 \times 10^{-5}$ & 17 & 0.16 & Yes & 0.2290 \\
$N 6$ & $2.0 \times 10^{-4}$ & 13 & 0.16 & Yes & 0.1094 \\
$N 7$ & $5.0 \times 10^{-4}$ & 9 & 0.16 & Yes & 0.0101 \\
\hline
\end{tabular}

